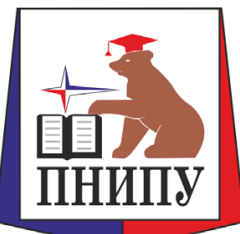

\title{
PNRPU MECHANICS BULLETIN
}

№ 3-4, 2018

http://vestnik.pstu.ru/mechanics/about/inf/

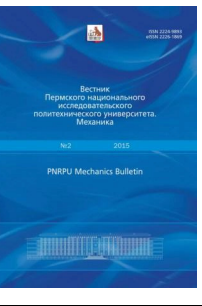

DOI: $10.15593 /$ perm.mech/eng.2018.3.12

UDC 539.4

\section{COMPLETE ASYMPTOTIC EXPANSION M. WILLIAMS NEAR THE CRACK TIPS OF COLLINEAR CRACKS OF EQUAL LENGTHS IN AN INFINITE PLANE MEDIUM}

\section{L.V. Stepanova, P.S. Roslyakov}

\author{
Samara State University, Samara, Russian Federation
}

\section{ARTICLE INFO}

Received: 11.07.2014

Accepted: 20.08.2014

Published: 25.12.2018

\section{Keywords:}

multiparametric description of the near crack tip stress filed, analytical solution, infinite plate with two collinear cracks, asymptotic expansion, amplitude coefficients of the complete asymptotic Williams expansions, higher-order terms.

\begin{abstract}
The paper is aimed at analytical determination of the coefficients in crack tip expansions for two collinear finite cracks of equal length in an infinite plane medium under mixed mode loading conditions. The study is based on the solutions of the complex variable theory in plane elasticity theory and the complete asymptotic Williams expansion of the stress field in the vicinity of the crack tip. From the practical point of view, it is very important to know: 1) analytical dependence of coefficients on geometrical parameters of specimens and applied loads; 2) the number of higherorder terms in the asymptotic Williams expansions that we need to keep to accurately describe the stress and displacement fields in the neighborhood of the crack tip. In the paper the authors have provided a multiparametric presentation of the stress filed near the crack tips in the infinite plate with two collinear cracks of finite length. We have presented the analytical determination method of coefficients related to the complete asymptotic expansion. The method is based on the complex variable theory and the classical Kolosoff-Muskhelishvili approach. The expansion of the KolosoffMuskhelishvili's potential in the vicinity of the crack tip allows to find the analytical presentation for coefficients of the complete Williams asymptotic expansion. The analytical solution gives the dependence of the amplitude coefficients on the lengths of the crack and the applied loads for pure mode I, pure mode II and mixed mode I/II conditions. It is shown that together with the main term including the stress intensity factor and the term called T-stress, it is necessary to hold higherorder terms in the asymptotic solution. The obtained solution permits to construct the asymptotic stress field expansion containing an arbitrary preassigned number of higher-order terms.
\end{abstract}




\section{Introduction. Asymptotic fields near the crack tip in the linear elastic material}

Describing the stress field in the vicinity of a crack, incision or a corner cut by means of an asymptotic expression containing high-order terms is an actual task of fracture mechanics $[1,2]$. Fracture mechanics has recently demonstrated [3-35], that the engineering applications of the traditional linear fracture mechanics, based on the only parameter, being the stress intensity factor, as the fault propagation criterion, require improvement and consideration of higher approximations in the complete asymptotic Williams expansion [39]. The multiparameter description of a stress field in the vicinity of a crack tip was suggested back in the year 1957 by M. Williams [39]. Since then, the solution of $\mathrm{M}$. Williams has been the classic solution of the linear fracture mechanics, where, however, in the most engineering applications only the first term was used, with the amplitude coefficient getting the title of the stress intensity factor. Only in the latest times, on the basis of the theoretical investigations, experimental works and computer simulation modelling [3-33], the fracture mechanics has formed a clear understanding of the need to hold the higher approximations within the complete asymptotic expression of the stress field in the vicinity of the crack tip.

The paper [3] review the researches dedicated to the assessment of T-stresses in the complete M. Williams solution in the vicinity of the crack tip. The authors describe various aspects of the impact made by T-stresses on the crack propagation trajectory, on the reproduction of the isochromatic lines picture, on the evaluation of the dimensions and the shape of the plastic yield area. The article discusses various methods of T-stress detection: experimental, analytical and numerical (finite element method). Authors provide a critical review of the existing researches and arrive at the conclusion that consideration of T-stress alone cannot ensure the accurate and reliable forecast of the crack propagation direction. For this reason, it requires the consideration of the high terms in the asymptotic expression of the stress field in the vicinity of the crack tip.

[4] discusses weak properties of the stress field in the vicinity of the crack tip (a typical example of which would be free edges of the laminate composite materials and the point lying in the joint lines of several materials) and the need for improved fracture criteria that would consider weaker singularity of the stress field. The work presents two models of crack formation at the junction of two materials.

Shlyannikov V.N. and Zakharov A.P. [5] made an experimental and numeric evaluation of the fatigue crack propagation rate under different systems of biaxial loading. $\mathrm{T}$-stresses were calculated for the $\mathrm{X}$-shaped specimens under mixed mode loading conditions. The mixed loading experiment analysis carried out by the authors is based on including the T-stresses into the computation model.
In [6] it is remarked that in many practical situations the contribution of high terms in the complete Williams expansion (besides the stress intensity factor and T-stresses) in engineering applications is not negligible. The main objective of [6] was to present equations that would accurately describe the stress fields in the vicinity of the crack tip, especially in the cases when the stress intensity factors for the opening mode cracks and transverse shear cracks combined with T-stresses cannot describe the stress distribution by the crack tip with the required accuracy. [6] studies a plane with a central crack under mixed mode loading conditions to demonstrated the need of holding the high terms. It is demonstrated that the deformation energy may be analytically computed with high accuracy provided that the four terms following the main asymptotic expansion member and T-stress are held.

[7] performs a systematic research of T-stresses and their contribution into the common stress field in the vicinity of the crack tip in a compact specimen. It studies the dependence of $\mathrm{T}$-stresses of the geometrical properties of the specimen. The objective of [8] is the determination of the stress intensity factors, T-stresses and three more terms in the complete asymptotic M. Williams expansion for two disc-shaped specimens in the complete diapason of mixed deformation forms. The work calculates the dimensionless functions of the factors of the singular, second and third members in the asymptotic stress expansions near the crack tip in disc-shaped specimens under mixed mode loading conditions (as a rule, they are defined $A_{3}, B_{3}$ for opening mode cracks and transverse shear cracks correspondingly). It is demonstrated that the amplitude scale coefficient in the third term is an important parameter characterizing the stress field near the crack tip.

In [9], the theoretical and experimental marble fracture research based on a number of specimens and mixed loading tests demonstrated that the high terms in complete asymptotic M. Williams expansion may make a significant impact on the assessment of rock resistance power. To determine the direction of crack propagation, the authors used the modified maximal tangential stress criterion that considered the non-singular terms in the asymptotic expansion as a component of stress tensor (T-stresses, $\left.A_{3}, B_{3}\right)$ in addition to the singular terms. The modified criterion was used for the analysis of triangular-shaped specimens. It was proved that the modified criterion considering the high approximations in the asymptotic $\mathrm{M}$. Williams expansion ensures a better compliance with the experimental data.

[10] notices the need of accurate analysis of the stress fields in the vicinity of the crack tip for a great number of engineering applications. The authors demonstrate that the higher-order terms in the asymptotic M. Williams expansion should not be neglected.

The objective of [11] is to describe the stress fields and shears in vicinity of the crack tip and at a distance from it in a number of experimental specimens for the determination 
of the fracture mechanics parameters. The article suggests specimens of new geometry for considering a wide scale of loading types and evaluating the dimensions of the nonlinear deformation areas occurring near the crack tip. For a four-point bending test, incised prismatic shapes were offered. Along with the experimental research, a numerical modeling for the same samples was done. In the asymptotic stress field expansion, the singular term, T-stress and higher approximations (non-singular terms) were held. The article compares the experimental results, the numerical analysis and the suggested asymptotic expansions depending on their distance to the crack tip, and the number of held terms in the asymptotic expansions. It demonstrates the efficiency of the suggested approach for the determination of the nonlinear deformation area size by the incision tip in the composite silicate material. Such materials are characterized with quasi-brittle behavior when fractured with the nonlinear softening zone by the crack tip. It has been stated that in the case of bending, the changes in the proportions of the geometric parameters of the tested specimens and the fulcrum position do not make a significant impact on the width of the non-linear area.

The work [12] presents the analysis of stress fields and shears by the crack tip in specimens with an edge crack used for penetration tests. In English literature, such specimens are referred to as WST-specimens. A multiparameter analysis of the stress field has been made, after which the non-singular terms were held in the asymptotic M. Williams expansion. The impact made by the edge conditions (specimen fixation conditions) on the stress intensity factor and the T-stresses has been studied. All in all, the analysis allows to consider up to 12 terms in the asymptotic expansion. The coefficients have been determined from the experimental data. The determined asymptotic expansion coefficients were used for the stress field reconstruction and further comparison with the computations based on the finite element method. The comparison clearly demonstrates that for long cracks and long distances from the crack tip, the asymptotic description of the stress field requires holding several terms in the complete asymptotic expansion of the stress field by the crack tip.

Article [13] considers three specimens, classic for fracture mechanics: a plate with one side incision, a place with a central crack in the uniaxial extension conditions and a plate with a central crack in the pure shear conditions. For describing the stress field by the crack tip, M. Williams asymptotic expression with held higher-order approximations was used. The amplitude expansion factors (scale coefficients) were determined from the finite element modeling results carried out with the multipurpose finiteelement program that allows studying specimen with various geometric proportions. The amplitude coefficients were found with the deterministic method suggested in [14], and the performed multiparameter analysis proves that one or even two M. Williams asymptotic expansion members are not enough. For acquiring reliable evaluations and for widening the asymptotic M. Williams expansion scope (for widening the asymptotic scope), the higher approximations shall be held.

The authors of [15] remark, that the main task in designing ceramic laminar materials is increasing the fracture energy of the "body - crack" system in the process of the energy release cased by the crack formation and its further propagation. For the ceramic composite materials, the occurrence of cracks in the composite layers and their bifurcate behavior is typical; for this reason, it is essential to forecast the direction of the crack propagation in modeling the ceramic composite materials. The experiments show that the second term in the asymptotic M. Williams expansion may be relevant in the description of the bifurcate behavior of a crack.

On the example of a bar with an edge incision exposed to a four-point bending test, the authors of [16] evaluate the impact of the higher terms in the complete asymptotic M. Williams expansion. The considered loading pattern may be used for practicing the loading of the cracked specimens and studying the various values of the mixed loading parameter. Relying on the Rankine criterion, the authors determine the plastic yield area configuration in the direct vicinity from the crack tip for the studied specimen. The article presents the computer modeling of loading of the described specimen with the finite element method in order to calculate the parameters of the complete asymptotic M. Williams expansion and to evaluate the geometry of the plastic yield area. The calculation results demonstrate that the higher approximations in the asymptotic expression of the stress field may be relevant, if the stretch of the nonlinear deformation area is large enough in comparison to the dimensions of the specimen. [17] presents the analytical presentations of the factors of the complete asymptotic expansion of the stress field by the finite crack tip in an infinite linear elastic plate. On the basis of the analytical solution of the elementary crack problem, the dependence of the scale coefficients on the crack length and system of applied loads was found for 1) an opening mode crack; 2) a pure transverse plane shear with an incision and 3) a mixed mode loading of a plate with a fault. $[18,19]$ discuss the issues of multi-parameter presentation of the stress field near the crack tip in an isotropic linear elastic material on the basis of the complete M. Williams expansion, and the accuracy of the deterministic method for the complete asymptotic expansion coefficient computation. The articles [20-28] are dedicated to various aspects of the polynomial asymptotic expansions of the stress field in the vicinity of the crack tip in linear elastic materials [21], in media with more compound rheology [22-28], and the issues of solving the related non-linear eigenvalue problems. [25] considers the solutions of the boundary problems obtained with regard to the higher approximations in the asymptotic presentations of the stress tensor components by the track tip for the cases of mixed deformation of specimens with faults.

[29] demonstrates the need for considering both singular and non-singular stress tensor components by the 
crack tip and the incision in the parametric and criterial problems of fracture mechanics. It discusses the spacial and two-dimensional models and criteria taking the T-stresses into account. Their use is demonstrated on the example of solving the problems of fracture mechanics including analysis of the stressed-deformed conditions in the vicinity of the crack tip, the experimental detection of crack resistance, the master-curve and the general diagram of crack resistance of bodies with cracks and incisions, forecasting of the mixed mode cracks propagation directions.

[30] presents the models and criteria of the fracture mechanics for the bodies with cracks, considering the nonsingular expansion terms of the stresses by the crack tip, developed for the analysis of fracture of the bodies with incisions in case of a normal fracture. It provides the criterial equations and ultimate curves for the fracture assessment. The authenticity of the suggested criterion is confirmed by the test results obtained on the specimen with through-cracks under various temperatures.

[31] presents the method of detecting the singular and non-singular components of the stress field in the neighborhood of the crack tip in the presence of the initial data error caused by the experimental or modeling inaccuracies. On the basis of the numeric experiments it was proved that the method of expansion of the tapered incision elasticity theory problem solution (asymptotic M. Williams solution) in eigenfunctions provides the opportunity of detecting the sought parameters with the accuracy consistent for engineering practice even in the presence of a significant error. The work presents the method of approximate assessment of the area where the modeling conditions of a plane elastic problem, caused by the presence of plastic deformation, geometric peculiarities or other factors, are not satisfied.

[33] suggests a method for detecting the stress and T-stress intensity factor for the opening mode crack on the basis of measuring the local deformation responses caused by the small increments of its length. The responses in the form of tangential displacement fields are registered with the electronic (digital) speckle interferometry. The experimental data interpretation is based on the complete asymptotic M. Williams expansion.

Matvienko Yu.G. considered [34] two possible models and criterial equations of the fracture mechanics of the bodies with incisions, considering the non-singular expansion terms by the incision (crack) tip and based on the crack resistance diagrams and the master curve. A criterial equation of the general crack resistance diagram, equally acceptable for a body with a crack and incision and considering the change in the deformation constraint by the incision tip as a result of the finiteness of the rounding radius of its tip and the non-singular component of the stresses ( $T$-stresses) is provided. The article introduces the definition of effective $T$-stresses, determined through averaging the $T$-stresses before the incision tip in the prefracture area, characterized with an effective distance.
The basic dependence of the fracture toughness in the function of the effective $T$-stresses (master curve) of pipe steel is built for the given incision geometry. [35] presents the promising models and criteria of the two-parameter fracture mechanics, considering the properties of the stressstrain behavior in the neighborhood of the crack-like fault tip. It draws the theoretical and experimental conclusions, allowing to assess the crack trajectory, construction strength and durability of the damaged critical elements with regard to the non-singular stress field component near the crack (incision) tip.

It can be concluded, therefore, that in the today's fracture mechanics there is an established clear understanding of the need of holding the high approximations in the complete asymptotic M. Williams expansion and the necessity for developing the methods and approaches for acquiring analytical dependences of the coefficients on the specimen geometry and the applied loading systems, and, therefore, for expanding the tables containing the found assessments for the stress intensity factors for various specimens, presentations for the higher term coefficients.

The present work studies the deformation of a plate with two collinear finite cracks of equal length, in the vicinity of which the asymptotic expressions of the stress field containing higher terms are built. It provides analytical expressions for the scale factors (coefficients) as functions of the crack length, crack tip coordinates and the applied load.

\section{Complete asymptotic M. Williams expansion of the stress field by the crack tip}

Complete asymptotic expression of the stress field in the neighborhood of the crack tip containing high-order terms, the asymptotic M. Williams solution, is the general description of the stress field near the crack tip [1, 2]. Asymptotic M. Williams expansion for each specimen configuration contains a sequence of coefficients depending on the geometric parameters of the specimen and the applied loading system. There are widely known analytical expressions for the first two terms in the asymptotic expansions of the stress field: for the stress intensity factors and $\mathrm{T}$-stresses for the great number of configurations of specimens with cracks $[1,17]$. Determination of the higher approximation coefficients as functions of the applied load and the geometry of the studied specimen with a crack is a more complicated task to solve. This work presents analytic expressions for the coefficients of the complete asymptotic M. Williams expansion of the stress field in the neighborhood of the tips of two collinear cracks in an infinite plane medium under mixed mode loading conditions, when the infinite medium is exposed to tension and shear load. The identification of the complete asymptotic M. Williams expansion coefficients is based on the precise analytic solution of the problem obtained with the classic comprehensive presentation of the plane 
elasticity theory problem solution [40, 41]. Using the eigenfunctions expansion method, M. Williams [39] presented Airy stress function in the neighborhood of the crack tip in the form $\chi(r, \theta)=\sum_{i} f_{i}(\theta) r^{\lambda_{i}}$, where $f_{i}(\theta)$ and $\lambda_{i}$ are eigenfunctions and eigenvalues correspondingly. At that, the stress tensor components in the neighborhood of the crack tip get the form

$$
\sigma_{i j}(r, \theta)=\sum_{m=1}^{2} \sum_{k=-\infty}^{\infty} a_{k}^{m} f_{k}^{m, i j}(\theta) r^{k / 2-1},
$$

where index $m$ corresponds to the load type $(m=1$ corresponds to the normal fracture, $m=2$ corresponds to transverse shear), coefficients $a_{k}^{m}$ depend on the geometry of the cracked specimens and the applied loading system, $f_{k}^{m, i j}(\theta)$ are the universal angular distributions determined in the process of the boundary problem solving. The angular stress distributions are well known [17] in (1):

$$
\begin{aligned}
& f_{k}^{1,11}(\theta)= k\left[\left(2+k / 2+(-1)^{k}\right) \cos (k / 2-1) \theta-\right. \\
&-(k / 2-1) \cos (k / 2-3) \theta] / 2, \\
& f_{k}^{1,22}(\theta)= k\left[\left(2+k / 2-(-1)^{k}\right) \cos (k / 2-1) \theta+\right. \\
&+(k / 2-1) \cos (k / 2-3) \theta] / 2, \\
& f_{k}^{1,12}(\theta)=k[(k / 2-1) \sin (k / 2-3) \theta- \\
&\left.-\left(k / 2+(-1)^{k}\right) \sin (k / 2-1) \theta\right] / 2, \\
& f_{k}^{2,11}(\theta)=-k\left[\left(2+k / 2-(-1)^{k}\right) \sin (k / 2-1) \theta-\right. \\
&-(k / 2-1) \sin (k / 2-3) \theta] / 2, \\
& f_{k}^{2,22}(\theta)=-k\left[\left(2-k / 2+(-1)^{k}\right) \sin (k / 2-1) \theta+\right. \\
&+(k / 2-1) \sin (k / 2-3) \theta] / 2, \\
& f_{k}^{2,12}(\theta)=k[(k / 2-1) \cos (k / 2-3) \theta- \\
&\left.-\left(k / 2-(-1)^{k}\right) \cos (k / 2-1) \theta\right] / 2 .
\end{aligned}
$$

Such properties of the crack mechanics boundary problems as the geometry of the incised area, the nature and intensity of the applied load do not constitute a part of either the radial or angular distribution of the stress tensor components. Therefore, the entire variety of fracture

mechanics problems is solved with the coefficients $a_{k}^{m}$. According to the definition, the stress intensity factor equal to

$$
K_{\mathrm{I}}=\lim _{r \rightarrow+0} \sqrt{2 \pi r} \sigma_{22}(r, 0), \quad K_{\mathrm{II}}=\lim _{r \rightarrow+0} \sqrt{2 \pi r} \sigma_{12}(r, 0) .
$$

Considering the (1-3) correlation, it is possible to find

$$
\begin{gathered}
K_{\mathrm{I}}=\sqrt{2 \pi} a_{1}^{1} f_{1}^{1,22}(0), \quad K_{\mathrm{II}}=\sqrt{2 \pi} a_{1}^{2} f_{1}^{2,12}(0), \\
T=a_{2}^{1} f_{2}^{1,11}(0) .
\end{gathered}
$$

By the present moment, fracture mechanics has developed a clear understanding of the need of holding the high-order terms in the complete asymptotic M. Williams expansion, and, therefore, of the necessity of determining its coefficients $a_{k}^{m}$ for various specimens frequently used in engineering practice [6-36]. One of the promising methods of finding the analytic expressions for the asymptotic expansion coefficients is the method based on the comprehensive stress field presentation - the KolosoffMuskhelishvili approach [40, 41] and the further merge of the analytic solution obtained with the complex variable function theory with the asymptotic solution of $\mathrm{M}$. Williams. The analytic presentations of the complete asymptotic M. Williams expansion coefficients for the infinite plate medium with two collinear cracks are shown below (Fig. 1).

\section{Comprehensive presentation of the plane medium with two collinear incisions loading problem solution}

The complex variable function theory provides a convenient mathematic tool for solving the plane elasticity problems. The approach initially suggested by Kolosoff [40] and developed by Muskhelishvili [41] is based on the introduction of the Airy stress function determined with the expressions

$$
\begin{gathered}
\sigma_{11}\left(x_{1}, x_{2}\right)=\frac{\partial^{2} \Phi}{\partial x_{2}^{2}}, \quad \sigma_{22}\left(x_{1}, x_{2}\right)=\frac{\partial^{2} \Phi}{\partial x_{1}^{2}}, \\
\sigma_{12}\left(x_{1}, x_{2}\right)=-\frac{\partial^{2} \Phi}{\partial x_{1} \partial x_{2}} .
\end{gathered}
$$

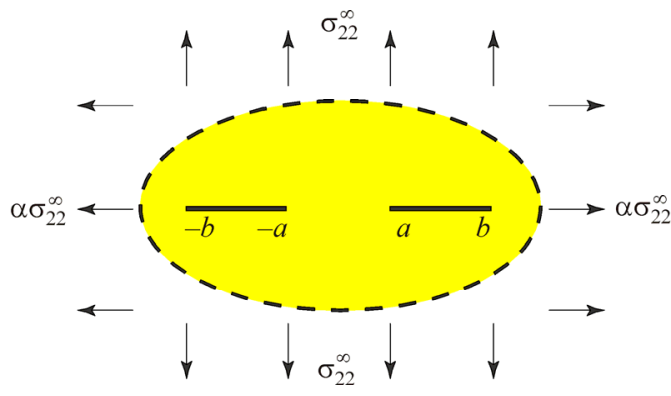

$a$

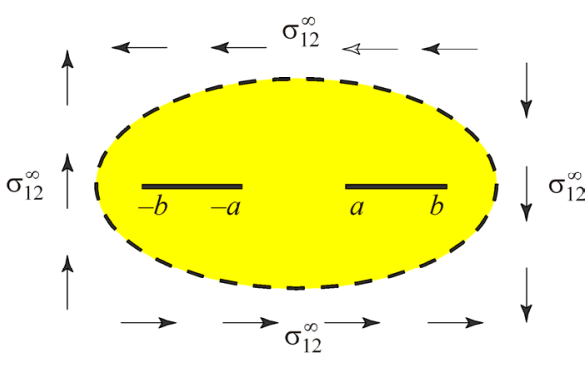

$b$

Fig. 1. Loading the plate with two collinear cracks of equal length: $a$ ) symmetric, $b$ ) asymmetric 
Following the Kolosoff-Muskhelishvili formalism, the biharmonic equation solution for the Airy stress function can be presented with the two complex potentials of the variable $z=x_{1}+i x_{2}$ :

$$
\Phi\left(x_{1}, x_{2}\right)=\operatorname{Re}[\bar{z} \varphi(z)+\chi(z)]
$$

where $\operatorname{Re}[]$ stands for the real part of the complex variable function. Combining the two latter correlations, it is possible to get the classic presentation of the stress tensor components

$$
\begin{gathered}
\sigma_{11}(z)+\sigma_{22}(z)=4 \operatorname{Re}\left[\varphi^{\prime}(z)\right], \\
\sigma_{22}(z)-\sigma_{11}(z)+2 i \sigma_{12}(z)=2\left[\bar{z} \varphi^{\prime \prime}(z)+\chi^{\prime}(z)\right] .
\end{gathered}
$$

Below, the complex solutions for the plate medium with two collinear cracks in the conditions of 1) normal fracture; 2) transverse shear; 3 ) mixed mode loading (normal fracture and transverse shear, index 1 corresponding to the normal fractures and 2 - to transverse shear) will be used. Since it is obvious that for a linear elastic isotropic medium the superposition principle is valid, the linear combination of solutions for the cases 1) and 2) provides an opportunity to get a comprehensive solution for mixed mode loading in the complete diapason of mixed forms of deformation of the plate with two collinear cracks.

For the normal fracture crack, the edge conditions on the infiniteness are formulated as follows

$$
\lim _{|z| \rightarrow+\infty}\left[\sigma_{11}(z), \sigma_{12}(z), \sigma_{22}(z)\right]=[\alpha, 0,1] \sigma_{22}^{\infty},
$$

for a transverse shear crack, the conditions in the point of infinity have the form of

$$
\lim _{|z| \rightarrow+\infty}\left[\sigma_{11}(z), \sigma_{12}(z), \sigma_{22}(z)\right]=[0,1,0] \sigma_{12}^{\infty} .
$$

In case of the biaxial symmetric strain of the plate (Fig. 1, a) the comprehensive stress field presentation has the form $[17,40,41]$

$$
\begin{gathered}
\sigma_{11}^{1}(z)=2 \operatorname{Re}\left[\varphi_{1}^{\prime}(z)\right]-2 x_{2} \operatorname{Im}\left[\varphi_{1}^{\prime \prime}(z)\right]+(\alpha-1) \sigma_{22}^{\infty} / 2, \\
\sigma_{22}^{1}(z)=2 \operatorname{Re}\left[\varphi_{1}^{\prime}(z)\right]+2 x_{2} \operatorname{Im}\left[\varphi_{1}^{\prime \prime}(z)\right]-(\alpha-1) \sigma_{22}^{\infty} / 2, \\
\sigma_{12}^{1}(z)=-2 x_{2} \operatorname{Re}\left[\varphi_{1}^{\prime \prime}(z)\right] .
\end{gathered}
$$

The complex potential $\varphi_{1}{ }^{\prime}(z)$ of the plate medium with two collinear cracks is determined with the formula [41]

$$
\varphi_{1}^{\prime}(z)=\frac{\sigma_{22}^{\infty}}{2} \frac{z^{2}-c}{\sqrt{\left(z^{2}-a^{2}\right)\left(z^{2}-b^{2}\right)}}+(\alpha-1) \frac{\sigma_{22}^{\infty}}{4},
$$

$c=b^{2} E(\pi / 2, k) / F(\pi / 2, k), \quad k=\sqrt{1-a^{2} / b^{2}}, \quad$ where $F(\pi / 2, k), \quad E(\pi / 2, k)$ are complete normal Legendre elliptic integral of the first kind. Expressions (4) with regard to (5) for the symmetric load of the crack (Fig. 1,a) get the form

$$
\begin{aligned}
& \sigma_{11}^{1}(z)=\sigma_{22}^{\infty}\left\{\operatorname{Re}\left[\frac{z^{2}-c}{\sqrt{\left(z^{2}-a^{2}\right)\left(z^{2}-b^{2}\right)}}\right]+(\alpha-1) \sigma_{22}^{\infty}-\right. \\
& \left.-x_{2} \operatorname{Im}\left[z \frac{2\left(z^{2}-a^{2}\right)\left(z^{2}-b^{2}\right)-\left(z^{2}-c\right)\left(2 z^{2}-a^{2}-b^{2}\right)}{\left(z^{2}-a^{2}\right)^{3 / 2}\left(z^{2}-b^{2}\right)^{3 / 2}}\right]\right\} \text {, } \\
& \sigma_{22}^{1}(z)=\sigma_{22}^{\infty}\left\{\operatorname{Re}\left[\frac{z^{2}-c}{\sqrt{\left(z^{2}-a^{2}\right)\left(z^{2}-b^{2}\right)}}\right]+\right. \\
& \left.+x_{2} \operatorname{Im}\left[z \frac{2\left(z^{2}-a^{2}\right)\left(z^{2}-b^{2}\right)-\left(z^{2}-c\right)\left(2 z^{2}-a^{2}-b^{2}\right)}{\left(z^{2}-a^{2}\right)^{3 / 2}\left(z^{2}-b^{2}\right)^{3 / 2}}\right]\right\} \\
& \sigma_{12}^{1}(z)=-\sigma_{22}^{\infty} x_{2}\left\{\operatorname { R e } \left[\frac{2 z}{\sqrt{\left(z^{2}-a^{2}\right)\left(z^{2}-b^{2}\right)}}-\right.\right. \\
& \left.\left.-\frac{z\left(z^{2}-c\right)\left(2 z^{2}-a^{2}-b^{2}\right)}{\left(z^{2}-a^{2}\right)^{3 / 2}\left(z^{2}-b^{2}\right)^{3 / 2}}\right]\right\} \text {. }
\end{aligned}
$$

For the transverse shear crack (Fig. 2, $b$ ), the stress field is determined with the complex potential $\varphi_{2}^{\prime}(z)$ :

$$
\begin{gathered}
\sigma_{11}^{2}(z)=2 \operatorname{Re}\left[\varphi_{2}^{\prime}(z)\right]-2 x_{2} \operatorname{Im}\left[\varphi_{2}^{\prime \prime}(z)\right], \\
\sigma_{22}^{2}(z)=2 x_{2} \operatorname{Im}\left[\varphi_{2}^{\prime \prime}(z)\right], \\
\sigma_{12}^{2}(z)=-2 \operatorname{Im}\left[\varphi_{2}^{\prime}(z)\right]-2 x_{2} \operatorname{Re}\left[\varphi_{2}^{\prime \prime}(z)\right]-C_{2} .
\end{gathered}
$$

In accordance with [41], the complex potential $\varphi_{2}^{\prime}(z)$ has the form

$$
\begin{gathered}
\varphi_{2}^{\prime}(z)=-i\left(\sigma_{12}^{\infty} / 2\right)\left[\left(z^{2}-c\right) / \sqrt{\left(z^{2}-a^{2}\right)\left(z^{2}-b^{2}\right)}\right]+i \sigma_{12}^{\infty} / 2 \\
C_{2}=\sigma_{12}^{\infty}
\end{gathered}
$$

The stress tensor components for the asymmetric loading of the crack get the form

$$
\begin{gathered}
\sigma_{11}^{2}(z)=\sigma_{12}^{\infty}\left\{2 \operatorname{Im}\left[\frac{z^{2}-c}{\sqrt{\left(z^{2}-a^{2}\right)\left(z^{2}-b^{2}\right)}}\right]+\right. \\
\left.+x_{2} \operatorname{Re}\left[z \frac{2\left(z^{2}-a^{2}\right)\left(z^{2}-b^{2}\right)-\left(z^{2}-c\right)\left(2 z^{2}-a^{2}-b^{2}\right)}{\left(z^{2}-a^{2}\right)^{3 / 2}\left(z^{2}-b^{2}\right)^{3 / 2}}\right]\right\} \\
\sigma_{22}^{2}(z)=-\sigma_{12}^{\infty} x_{2} \operatorname{Re}\left[\frac{2 z}{\sqrt{\left(z^{2}-a^{2}\right)\left(z^{2}-b^{2}\right)}}-\right. \\
\left.-\frac{z\left(z^{2}-c\right)\left(2 z^{2}-a^{2}-b^{2}\right)}{\left(z^{2}-a^{2}\right)^{3 / 2}\left(z^{2}-b^{2}\right)^{3 / 2}}\right]
\end{gathered}
$$




$$
\begin{gathered}
\sigma_{12}^{2}(z)=\sigma_{12}^{\infty}\left\{\operatorname{Re}\left[\frac{z^{2}-c}{\sqrt{\left(z^{2}-a^{2}\right)\left(z^{2}-b^{2}\right)}}\right]-\right. \\
\left.-x_{2} \operatorname{Im}\left[z \frac{2\left(z^{2}-a^{2}\right)\left(z^{2}-b^{2}\right)-\left(z^{2}-c\right)\left(2 z^{2}-a^{2}-b^{2}\right)}{\left(z^{2}-a^{2}\right)^{3 / 2}\left(z^{2}-b^{2}\right)^{3 / 2}}\right]\right\} .
\end{gathered}
$$

Fig. 2-4 demonstrates the lines of equal levels of the stress tensor components and the stress intensity in the plate medium with two collinear cracks of equal length for different types of mixed mode loading in the full diapason of loads characterized with the promiscuity parameter $M^{e}=(2 / \pi) \operatorname{arctg}\left(\sigma_{22}^{\infty} / \sigma_{12}^{\infty}\right):$ from normal fracture $\left(M^{e}=1\right)$
(Fig. 2, a) to transverse shear $\left(M^{e}=0\right)$ (Fig. 4, a). The diagrams are constructed with the formulae (6) for normal fracture, (8) for transverse shear and superposition of solutions (6) and (8) for mixed mode loading. The geometric parameters of the collinear cracks equal to $a /(2 l)=1, b /(2 l)=2$, where $l$ is the crack half-length.

Fig. $4, b$ shows the level lives of the stress tensor component for closer located cracks: for the following values of the geometric parameters $a /(2 l)=0.25, b /(2 l)=1.25$. The figures show that at $a /(2 l)=0.25, b /(2 l)=1.25$ the cracks make a stronger impact on each other in comparison to the previously considered geometric parameters $a /(2 l)=1, b /(2 l)=2$.

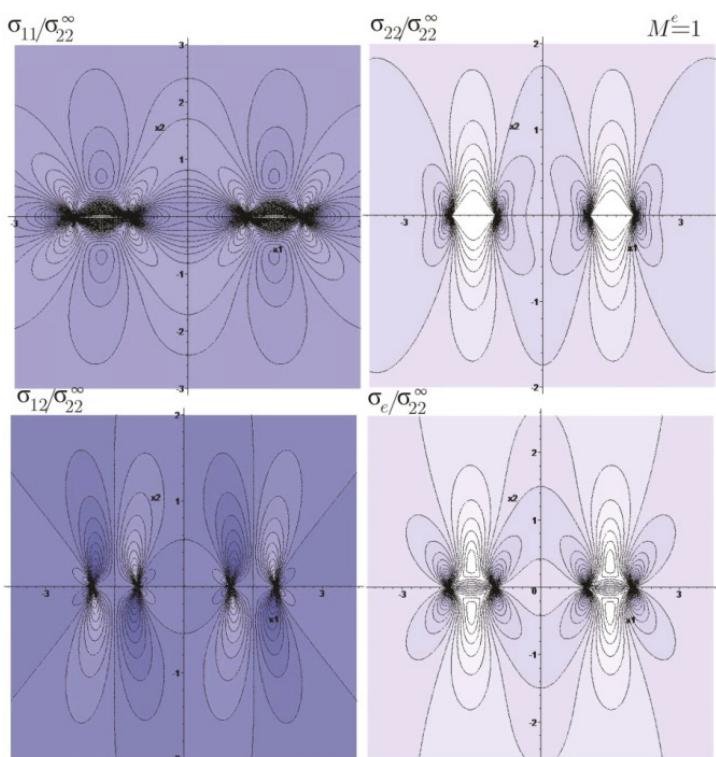

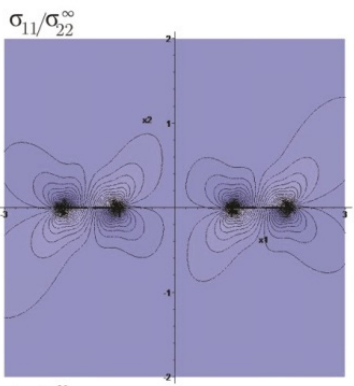

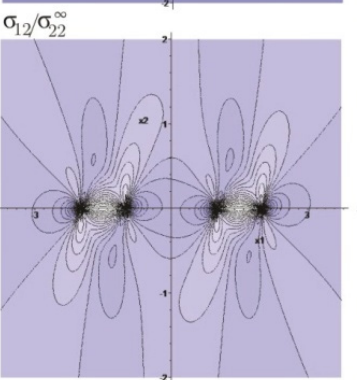

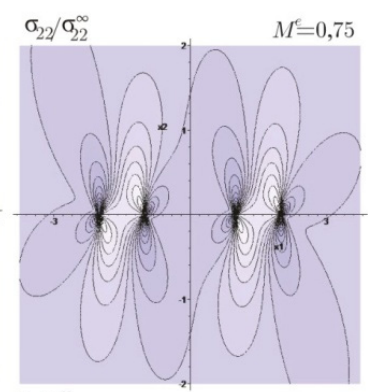

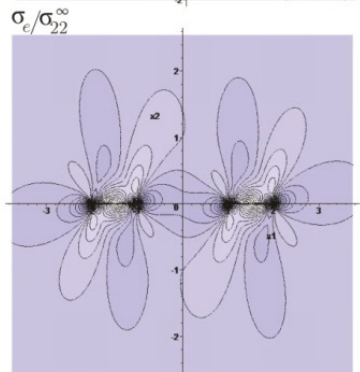

$b$

Fig. 2. The level lines of the stress tensor components and stress intensity for the plate medium with two collinear cracks of equal length: a) normal fracture; $b$ ) loading complying with the loading promiscuity parameter $M^{e}=0.75$
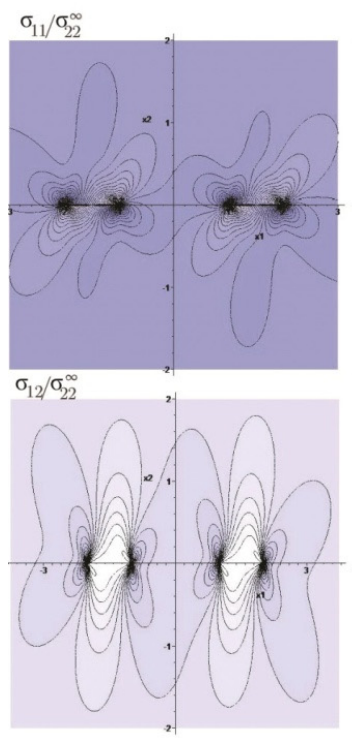

a
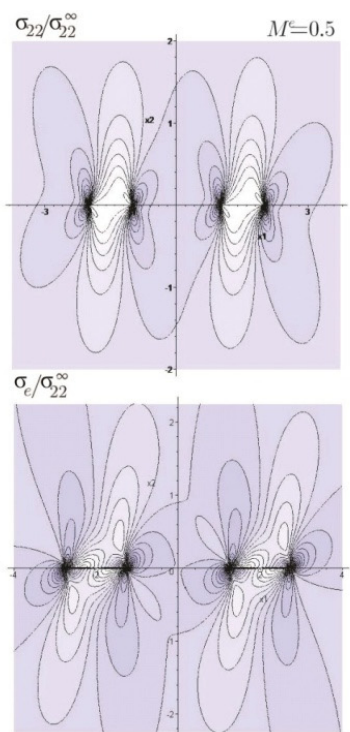
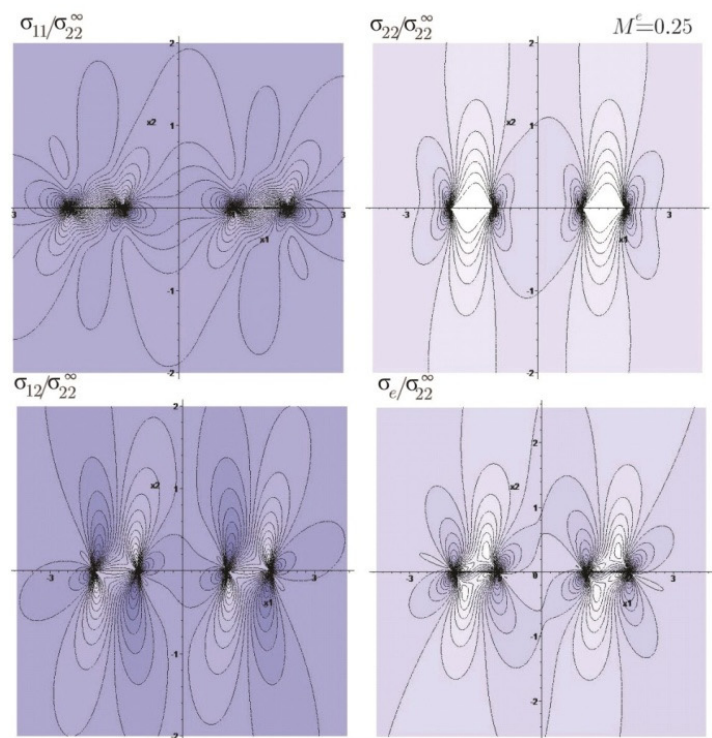

Fig. 3. The level lines of the stress tensor components and stress intensity (mixed mode loading of a plate medium with two collinear cracks of equal length): a) mixed mode loading complying with $M^{e}=0.5 ; b$ ) mixed mode loading complying with $M^{e}=0.25$ 

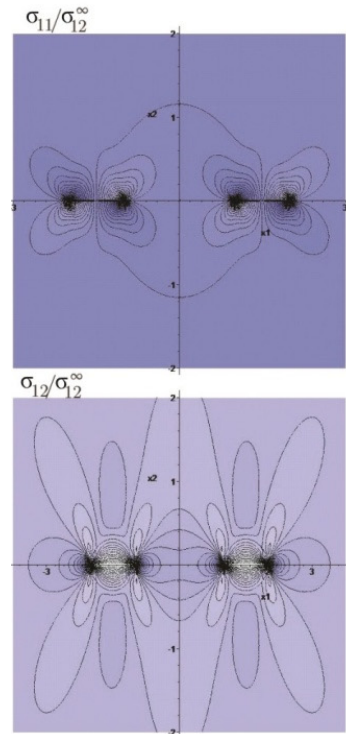

$a$
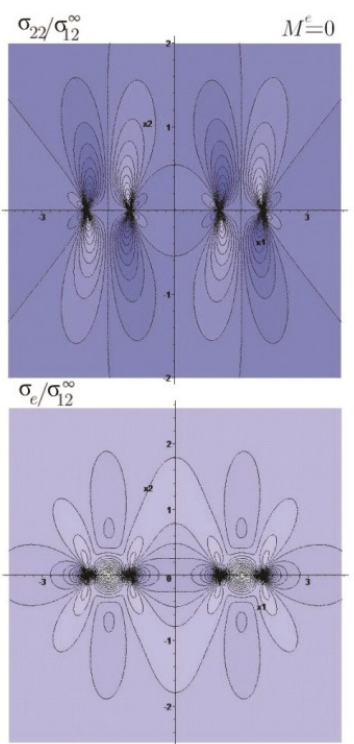
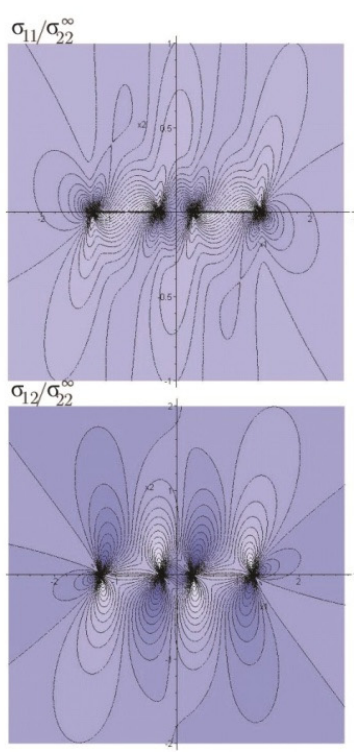

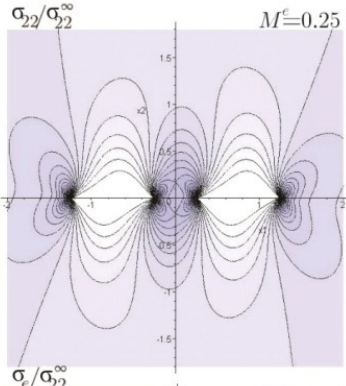

$\sigma_{e} / \sigma_{22}^{\infty}$

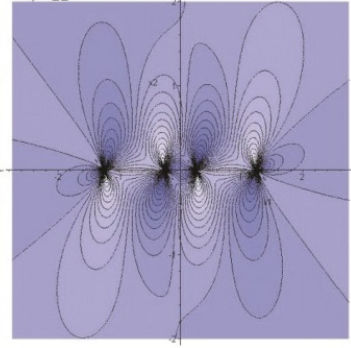

$b$

Fig. 4. Asymmetric loading of a plate medium with two collinear cracks of equal length (transverse shear) (a) and mixed loading of a plate for close cracks $(b)$

Comparing the asymptotic solution of M. Williams with the angular distributions (1) and (5), it is possible to find the stress intensity factor $K_{\mathrm{I}}(b)=$ $=\sqrt{\pi} \sigma_{22}^{\infty} b^{3 / 2}[1-E(\pi / 2, k) / F(\pi / 2, k)] / \sqrt{b^{2}-a^{2}}$ and the T-stress $T=(\alpha-1) \sigma_{22}^{\infty}$ or in a more general form for the transverse shear and axial separation cracks ( $2 l$ being the crack length and $d$ being the distance between the middles of two cracks)

$$
\left\{K_{\mathrm{I}}(b), K_{\mathrm{II}}(b), K_{\mathrm{III}}(b)\right\}=G(b)\left\{\sigma_{22}^{\infty}, \sigma_{12}^{\infty}, \sigma_{23}^{\infty}\right\} \sqrt{\pi l},
$$

where

$$
G(b)=\frac{d+2 l}{4 l}\left(\frac{d+2 l}{d}\right)^{1 / 2}\left[1-\frac{E(k)}{F(k)}\right] .
$$

Similarly the stress intensity factors can be found for another crack tip

$$
\left\{K_{\mathrm{I}}(a), K_{\mathrm{II}}(a), K_{\mathrm{III}}(a)\right\}=G(a)\left\{\sigma_{22}^{\infty}, \sigma_{12}^{\infty}, \sigma_{23}^{\infty}\right\} \sqrt{\pi l},
$$

where

$$
\begin{gathered}
G(a)=\frac{d-2 l}{4 l}\left(\frac{d-2 l}{d}\right)^{1 / 2}\left[\left(\frac{d+2 l}{d-2 l}\right)^{2} \frac{E(k)}{F(k)}-1\right], \\
l=(b-a) / 2, d=a+b .
\end{gathered}
$$

\section{Analytic Determination of the Complete Asymptotic M. Williams Expansion Coefficients of the Stress Field for the Normal Fracture Crack Tip}

The asymptotic expansion of a complex potential (5) in the neighborhood of the crack tip $z(r, \theta)=b+r e^{i \theta}$, $x_{2}=r \sin \theta$ has the form

$$
\varphi_{1}^{\prime}(z)=\frac{\sigma_{22}^{\infty}}{2} \sum_{n=0}^{\infty} q_{n}(z-b)^{n-1 / 2}+(\alpha-1) \frac{\sigma_{22}^{\infty}}{4},
$$

where

$$
\begin{gathered}
q_{n}=\sum_{k=0}^{n+1} p_{k} d_{n-k}, \quad p_{n}=\sum_{k=0}^{n+1} c_{k} e_{n-k}, \quad e_{n}=\sum_{k=0}^{n+1} a_{k} b_{n-k}, \\
d_{0}=b^{2}-c, \quad d_{1}=2 b, \quad d_{2}=1, \quad d_{k}=0, k>2, \\
a_{k}=(-1)^{k} \frac{(|2 k-1| ! !)}{2^{k} k !}(b-a)^{-(2 k+1) / 2}, \\
b_{k}=(-1)^{k} \frac{(|2 k-1| ! !)}{2^{k} k !}(a+b)^{-(2 k+1) / 2}, \\
c_{k}=(-1)^{k} \frac{(|2 k-1| ! !)}{2^{k} k !}(2 b)^{-(2 k+1) / 2} .
\end{gathered}
$$

Formulae (10)-(13) contain the dependence of the asymptotic expansion coefficients of the complex potential on the geometric parameters of the specimen $a, b$ and the applied strain load $\sigma_{22}^{\infty}$. Substituting the expression (9) into the Kolosoff-Muskhelishvili formulae (5) and comparison with the complete asymptotic M. Williams expansion (1) leads to the determination of the amplitude coefficients of the expansion:

$$
\begin{gathered}
a_{k}^{1}=\sigma_{22}^{\infty} q_{k} / f_{k}^{1,11}(\theta=0), \\
a_{2}^{1}=(\alpha-1) \sigma_{22}^{\infty} / 4, \quad a_{2 k}^{1}=0, \forall k>1 .
\end{gathered}
$$

Below, the analytic expressions for the first thirteen coefficients of the complete asymptotic M. Williams expansion, obtained with the expressions (10)-(13), are presented: 


$$
\begin{gathered}
a_{1}^{1}=\frac{\sigma_{22}^{\infty}}{\sqrt{2 b}} \frac{b^{2}-c}{\sqrt{b^{2}-a^{2}}}, a_{2}^{1}=\frac{(\alpha-1)}{4} \sigma_{22}^{\infty}, \quad a_{2 k}^{1}=0, \forall k>1, \\
a_{3}^{1}=\frac{\sigma_{22}^{\infty} \sqrt{2}}{24} \frac{3 b^{4}-7 a^{2} b^{2}+5 b^{2} c-a^{2} c}{b^{3 / 2}\left(b^{2}-a^{2}\right)^{3 / 2}}, \\
=-\frac{\sigma_{22}^{\infty} \sqrt{2}}{320} \frac{2 a^{2} b^{2} c-34 a^{2} b^{4}-19 a^{4} b^{2}+43 b^{4} c+5 b^{6}+3 a^{4} c}{b^{5 / 2}\left(b^{2}-a^{2}\right)^{5 / 2}}, \\
\times\left(-5 a^{6} c+137 a^{2} b^{4} c+11 a^{4} b^{2} c+177 b^{6} c+\right. \\
\left.+13 a^{6} b^{2}+7 b^{8}-113 a^{2} b^{6}-227 a^{4} b^{4}\right) /\left(b^{7 / 2}\left(b^{2}-a^{2}\right)^{7 / 2}\right), \\
\left.-124 a^{6} b^{2} c-67 a^{8} b^{2}-132 a^{6} b^{4}-1636 a^{2} b^{8}-7170 a^{4} b^{6}\right\},(14) \\
\times\left\{45 b^{10}+5732 a^{2} b^{6} c+450 a^{4} b^{4} c+2867 b^{8} c+35 a^{8} c-\right. \\
a_{92}^{1} \sqrt{2} \\
a_{9}^{1}=-\frac{\sigma_{22}^{\infty} \sqrt{2}}{36864} \frac{1}{b^{9 / 2}\left(b^{2}-a^{2}\right)^{9 / 2}} \times
\end{gathered}
$$

$\times\left\{10398 a^{4} b^{6} c+42997 a^{2} b^{8} c+77 b^{12}-10986 a^{6} b^{6}-\right.$

$$
-367 a^{8} b^{4}-646 a^{6} b^{4} c+295 a^{8} b^{2} c+11531 b^{10} c-
$$

$$
\begin{gathered}
\left.-6189 a^{2} b^{10}-47150 a^{4} b^{8}-63 a^{10} c+103 a^{10} b^{2}\right\}, \\
a_{13}^{1}=\frac{\sigma_{22}^{\infty} \sqrt{2}}{1703936} \frac{1}{b^{13 / 2}\left(b^{2}-a^{2}\right)^{13 / 2}} \times \\
\times\left\{48014 a^{2} b^{12}+559049 a^{4} b^{10}+329124 a^{6} b^{8}+11729 a^{8} b^{6}-\right. \\
-92479 b^{12} c-1810 a^{10} b^{4}-273 b^{14}+343 a^{12} b^{2}- \\
-551534 a^{2} b^{10} c+1330 a^{10} b^{2} c- \\
\left.-298169 a^{4} b^{8} c-1764 a^{6} b^{6} c-3329 a^{8} b^{4} c-231 a^{12} c\right\} .
\end{gathered}
$$

Substituting the asymptotic presentation for the complex potential (9) into (4), it is possible to acquire the asymptotic presentations of the stress tensor components in vicinity of the crack tip $z=b$

$$
\sigma_{i j}^{1}(r, \theta)=\sigma_{22}^{\infty}\left[\sum_{n=0}^{\infty} q_{n} g_{n}^{1, i j}(\theta) r^{n-1 / 2}+(\alpha-1) \delta_{i 1} \delta_{j 1}\right],
$$

where the angular distributions $g_{n}^{m, i j}(\theta)$ are determined as

$$
g_{n}^{1,11}(\theta)=
$$

$[(n+3 / 2) \cos (n-1 / 2) \theta-(n-1 / 2) \cos (n-5 / 2) \theta] / 2$,

$$
g_{n}^{1,22}(\theta)=
$$

$=[(-n+5 / 2) \cos (n-1 / 2) \theta+(n-1 / 2) \cos (n-5 / 2) \theta] / 2$,

$$
g_{n}^{1,12}(\theta)=
$$

$=[(-n+1 / 2) \sin (n-1 / 2) \theta+(n-1 / 2) \sin (n-5 / 2) \theta] / 2$.

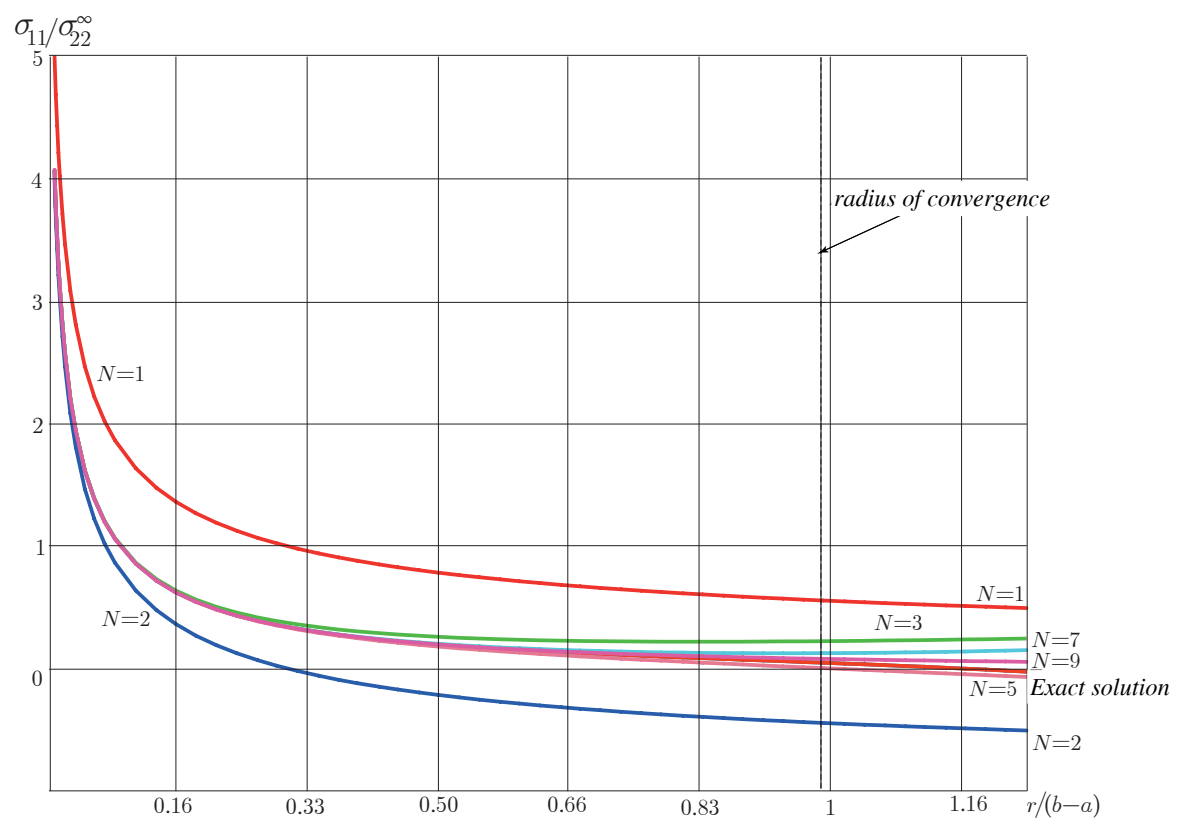

Fig. 5. Radial distribution of the component $\sigma_{11}^{1}$ near the crack tip $z=b$, constructed with the exact solution and the $k$-nomial asymptotic expansion, where $k=1,2,3,4,5,6,7,8,9$ 


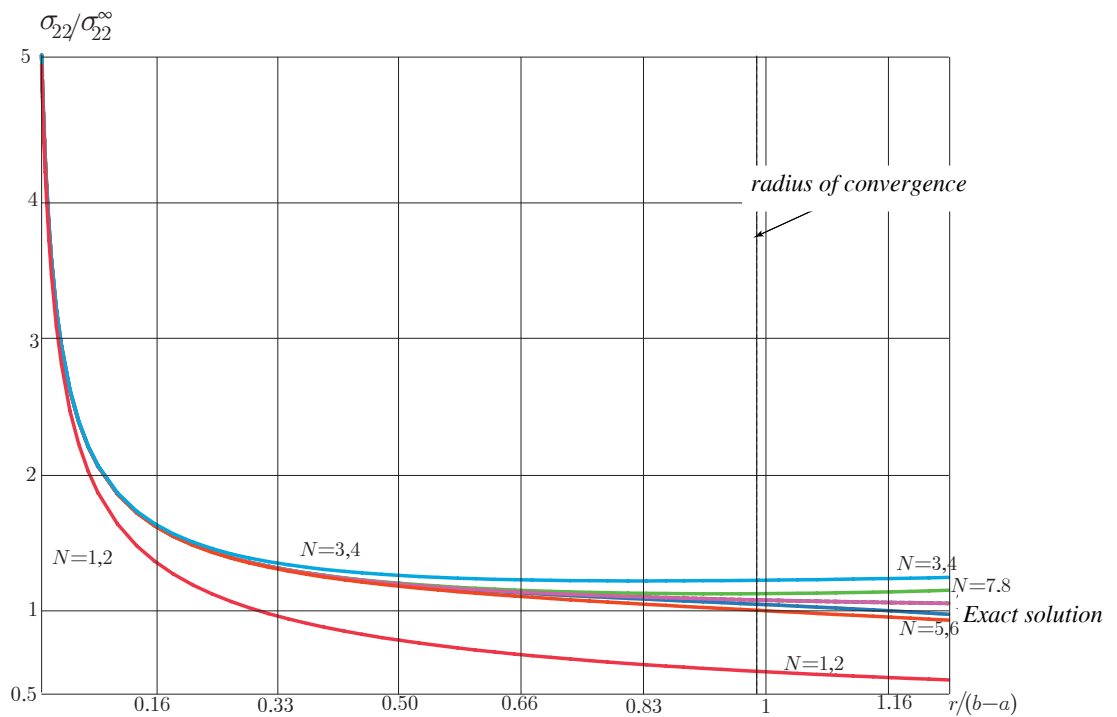

Fig. 6. Radial distribution of the component $\sigma_{22}^{1}$ near the crack tip $z=b$, constructed with the exact solution and the $k$-nomial asymptotic expansion, where $k=1,2,3,4,5,6,7,8,9$

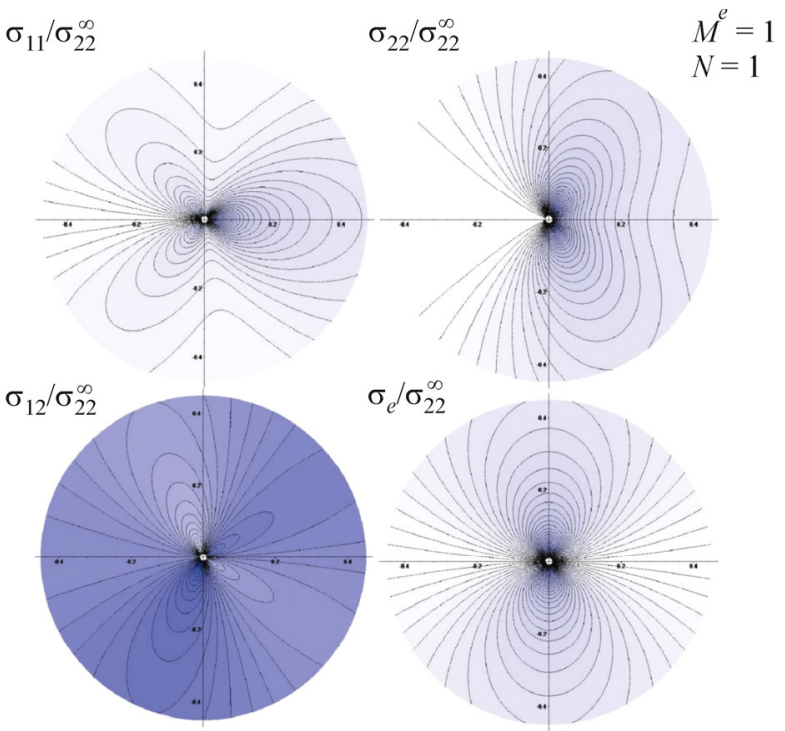

$a$

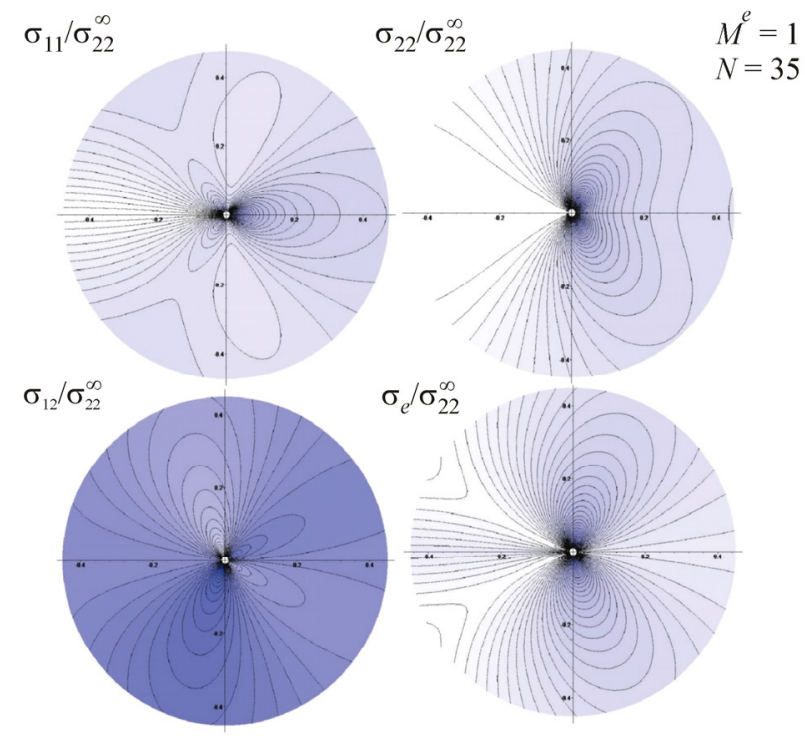

$b$

Fig. 7. Distribution of the stress tensor components and the stress intensity (level lines) in the vicinity of the tip $z=b$ for the fracture crack, built with the mononomial asymptotic expansions $(a)$ and thirty-five-nomial asymptotic expansions $(b)$

Fig. 5, 6 demonstrate the radial distributions of the normal stress tensor components $\sigma_{11}^{1}$ and $\sigma_{22}^{1}$ near the crack tip $z=b$, constructed with the exact solution and the $k$-nomial asymptotic expansions, where $k=1,2,3,4,5,6,7,8,9$. Fig. 5, 6 show that the T-stress makes a significant contribution into the asymptotic expansion of the stress tensor component $\sigma_{11}^{1}$. Moreover, Fig. 5, 6 lead to the conclusion that it is wrong to focus on the T-stress only, as the binomial asymptotic expansion of the considered stress tensor components is significantly different from the radial distribution, constructed on the basis of the exact solution. The presented figures demonstrate that the curves, determined with the asymptotic expansions containing the
9 terms, coincide with the exact solution (Fig. 5, 6). Fig. 7, 8 show the equal level lines of the stress tensor components $\sigma_{i j}^{1}$ and the shearing strain intensity in the neighborhood of the crack tip $z=b$, built with the mononomial (Fig. 7, $a$ ) and thirty-five-nomial asymptotic expansions (Fig. 7, b). Comparison Fig. 7, $a$ and $b$ shows that the level lines change and the higher approximation are critical for consideration. Fig. 8 demonstrates the intensity level lines of the shearing strain intensity in the neighborhood of the tip $z=b$ for the fracture crack, constructed with the main member of the asymptotic expansion (Fig. 8, $a$ and $b$ ) and the thirty-five-nomial asymptotic expansion (Fig. 8, c). Level lines in Fig. 8, $a$ and $b$ are obtained for different 
distances from the crack tip. Fig. 8, $c$ shows that the complete asymptotic M. Williams expansion, constructed with consideration of the higher approximations in the neighborhood of the tip $z=b$ has a greater fairness scope, as it "detects" the presence of the tip $z=a$. However for constructing the complete picture of the level lines of stress tensor components, a compound expansion, built with consideration of the asymptotic stress field both in the vicinity of the tip $z=b$, and in the vicinity of the tip $z=a$, the determination of which is an independent task not covered by this research, is required.

Fig. 9-12 present the angular distributions of the stress tensor components $\sigma_{11}^{1}$ in the vicinity of the fracture crack tip in different distances from the crack tip. Fig. 9, $a$ show the angular distributions in the distance $\hat{r}=r / a=0.075$. Fig. 9 demonstrates that the angular distribution determined with the mononomial asymptotic expansion (red curve) significantly differs from the angular distribution built with the binomial expansion (blue curve). However, the curves built with the trinomial, five-nomial and $k$-nomial asymptotic expansion of the stress field are not distinguishable. Therefore, in the given distance from the crack tip it is enough to hold three members in the asymptotic M. Williams expansion. If it is necessary to expand the asymptotic work scope $(1,2)$, then higher approximations shall remain in the asymptotic presentation. For example, Fig. 9, $b$ shows the angular distributions of the stress tensor components $\sigma_{11}^{1}$ in the distance $\hat{r}=r / a=0.15$. The trinomial asymptotic M. Williams expansion becomes distinguishable from the five-nomial expansion (green and violet curves in the Figure). The curves built with the seven-nomial, nine-nomial and $k$-nomial expansions are not distinguishable. Further increase of the distance from the crack tip causes the need of holding the following terms in the asymptotic presentation (Fig. 10, 11). Fig. 12, $a, b$ shows the angular distributions of the stress tensor components under $\hat{r}=r / a=0.5$, built with the held terms of different numbers in the asymptotic expansion, starting from the mononomial asymptotic expansion and finishing with the asymptotic expansion of 50 terms. Fig. 12 shows that the curves stop being different from each other only starting from the asymptotic expansion of 15 terms.

Therefore to expand the scope of the stress field asymptotic expansion in the vicinity of the crack tip, it is required to hold the higher approximations.

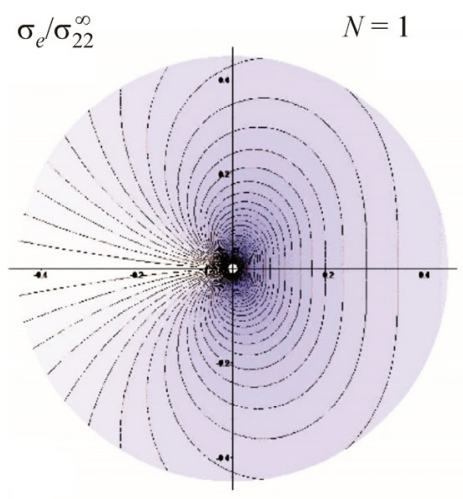

$a$

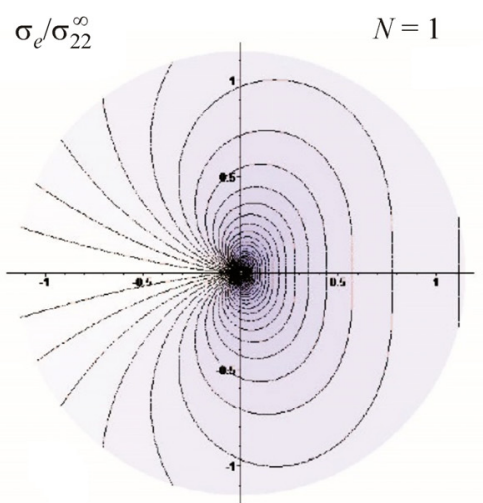

$b$

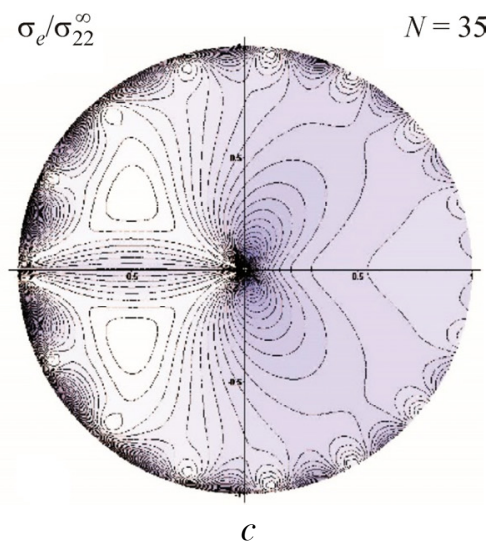

Fig. 8. Distribution of the stress intensity (level lines) in the vicinity of the tip $z=b$ for the fracture crack, built with a mononomial asymptotic expansion $(a)$ and for greater distances from the crack tip $(b)$ and the thirty-five-nomial asymptotic expansion $(c)$
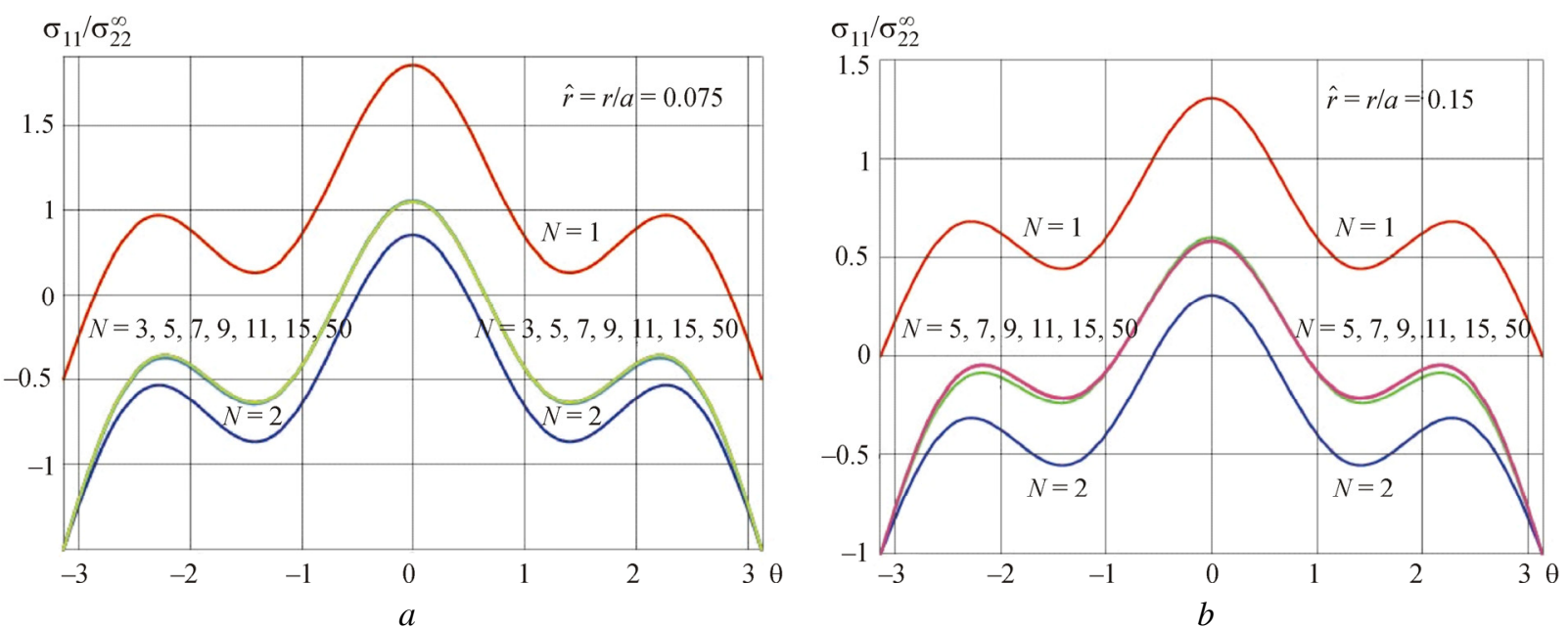

Fig. 9. The angular distribution of the stress tensor component $\sigma_{11}^{1}$ in the neighborhood of the crack tip $z=b$ in different distances from the crack tip: $a$ ) for $\hat{r}=r / a=0.075 ; ; b)$ for $\hat{r}=r / a=0.15$ 

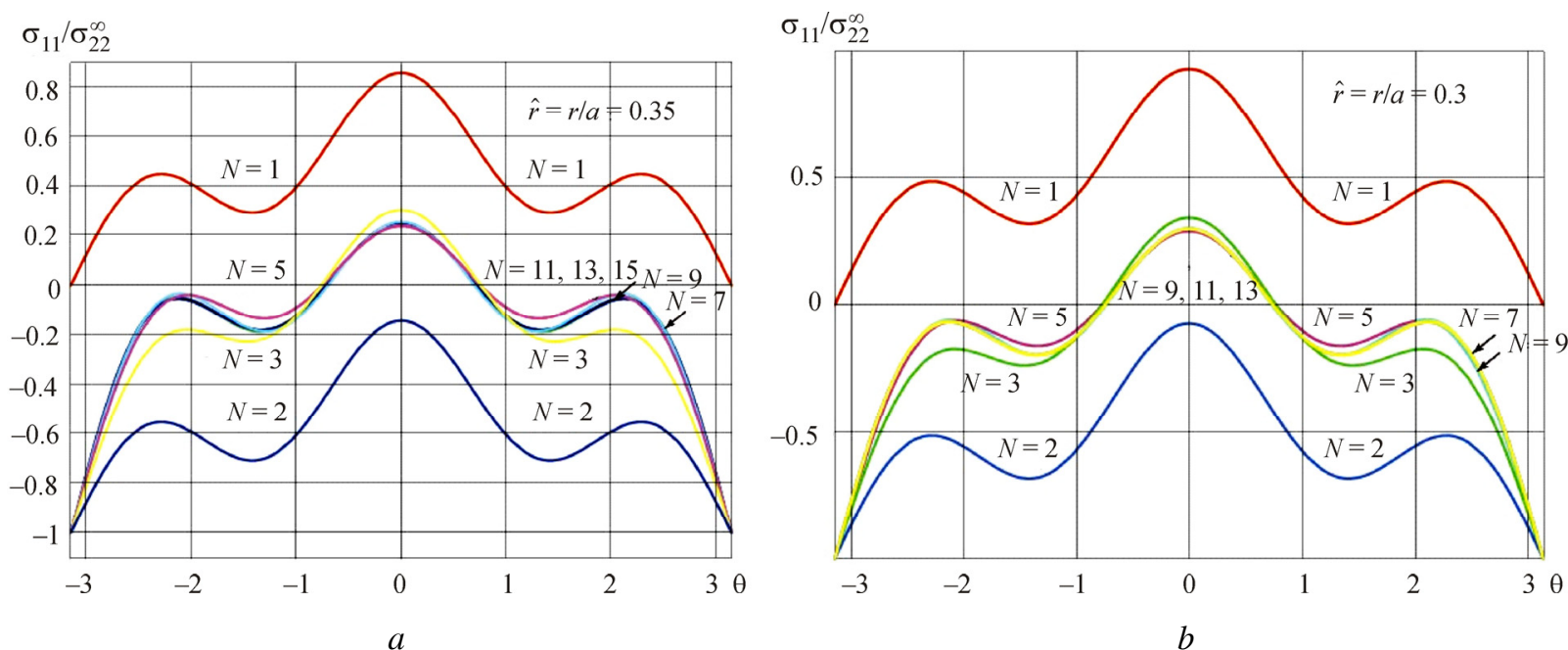

Fig. 10. The angular distribution of the stress tensor component $\sigma_{11}^{1}$ in the neighborhood of the crack tip $z=b$ in different distances from the crack tip: $a$ ) for $\hat{r}=r / a=0.3 ; b$ ) for $\hat{r}=r / a=0.35$
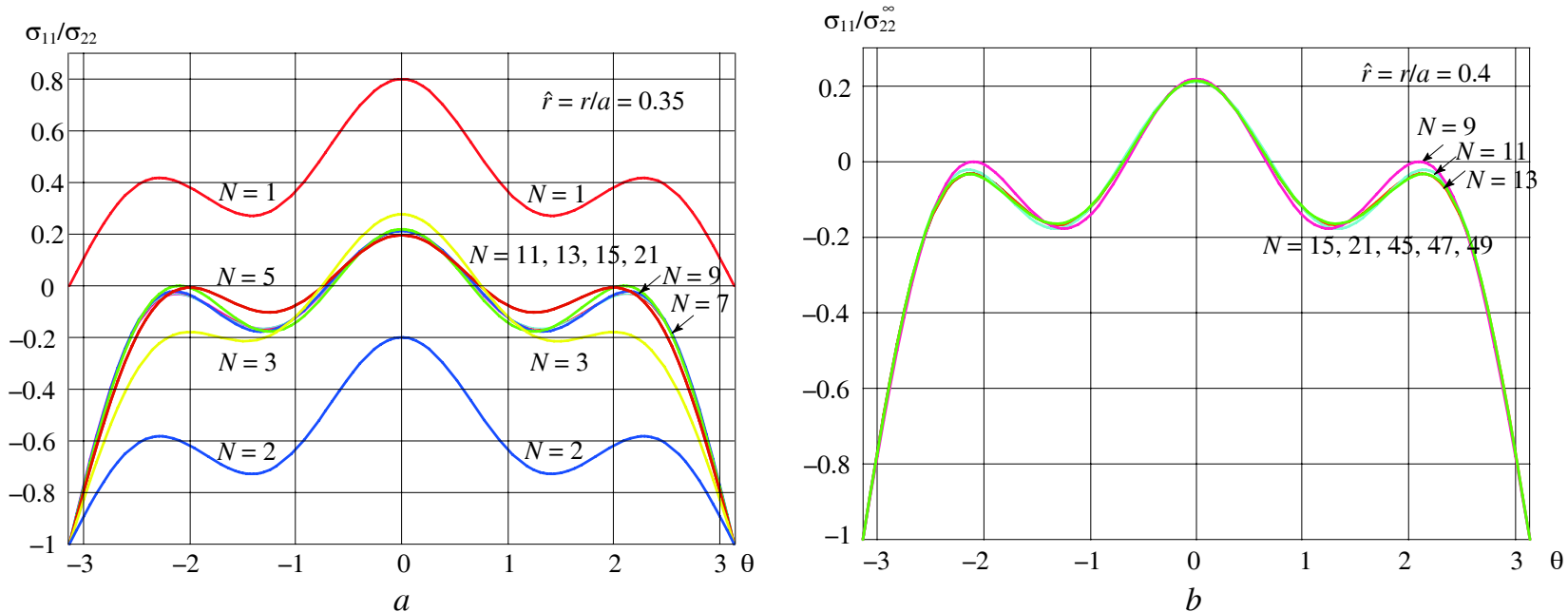

Fig. 11. The angular distributions of the stress tensor component $\sigma_{11}^{1}$ in the neighborhood of the crack tip $z=b$ for $\hat{r}=r / a=0.4$, built with the higher approximations in the asymptotic expansion
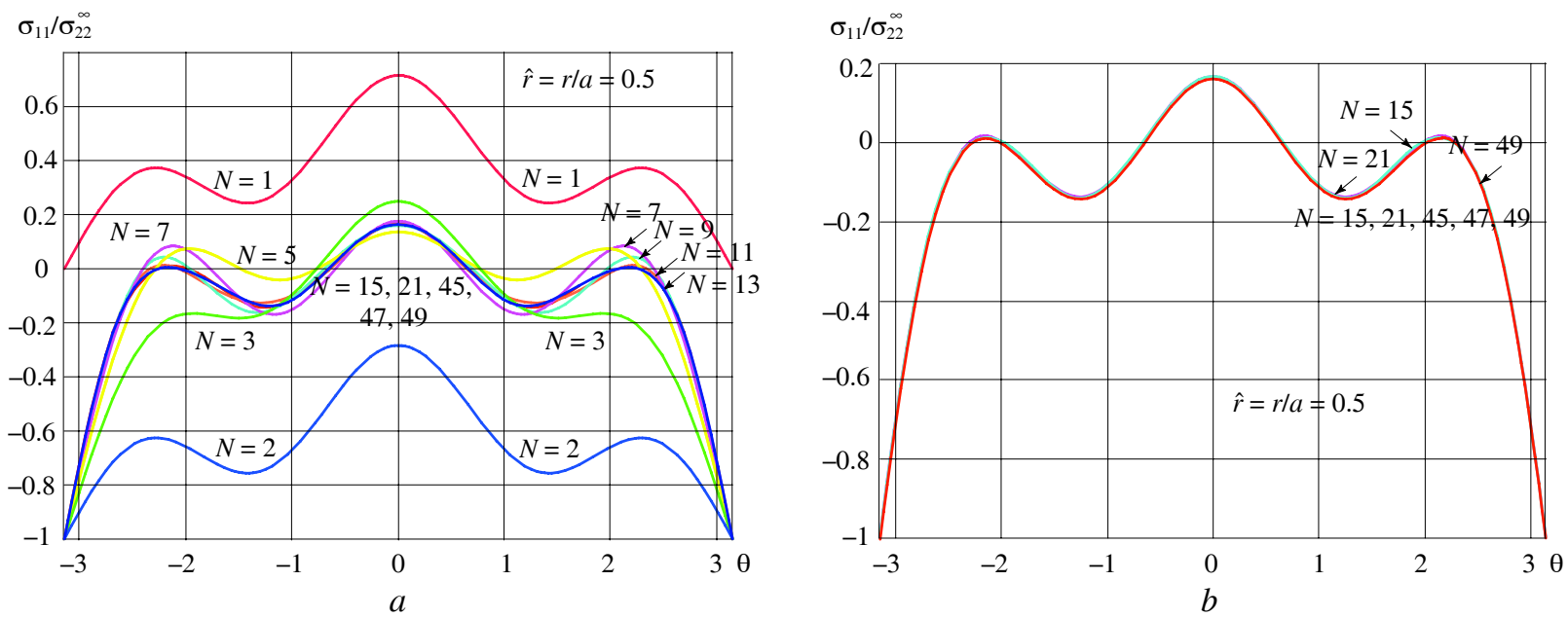

Fig. 12. The angular distributions of the stress tensor component $\sigma_{11}^{1}$ in the neighborhood of the crack tip $z=b$, built with the higher approximations in the asymptotic expansion 
Similarly, it is possible to obtain the complete asymptotic expansion in the vicinity of the tip $z=a$. Expanding the complex potential in a Taylor's series in the vicinity of the tip $z=a+r e^{i \theta}$, it is possible to find

$$
\varphi_{1}^{\prime}(z)=\frac{\sigma_{22}^{\infty}}{2} \sum_{n=0}^{\infty} \delta_{n}(z-a)^{n-1 / 2}+(\alpha-1) \frac{\sigma_{22}^{\infty}}{4},
$$

where

$$
\begin{gathered}
\delta_{n}=\sum_{k=0}^{n+1} \alpha_{k} \chi_{n-k}, \quad \chi_{n}=\sum_{k=0}^{n+1} \beta_{k} \xi_{n-k}, \quad \xi_{n}=\sum_{k=0}^{n+1} \varsigma_{k} \eta_{n-k}, \\
\eta_{0}=a^{2}-c, \quad \eta_{1}=2 a, \quad \eta_{2}=1, \quad \eta_{k}=0, k>2, \\
\alpha_{k}=(-1)^{k} \frac{(|2 k-1| ! !)}{2^{k} k !}(2 a)^{-(2 k+1) / 2}, \\
\beta_{k}=\frac{(|2 k-1| ! !)}{2^{k} k !}(b-a)^{-(2 k+1) / 2}, \\
\varsigma_{k}=(-1)^{k} \frac{(|2 k-1| ! !)}{2^{k} k !}(a+b)^{-(2 k+1) / 2} .
\end{gathered}
$$

Formulae (16)-(19) contain the dependence of the asymptotic expansion coefficients of the complex potential on the geometric parameters of the specimen $a, b$ and the applied strain load $\sigma_{22}^{\infty}$. Substituting the expression (15) into the Kolosoff-Muskhelishvili formulae (5) and comparison with the complete asymptotic M. Williams expansion (1) leads to the determination of the amplitude coefficients of the expansion:

$$
a_{k}^{1}=\sigma_{22}^{\infty} q_{k} / f_{k}^{1,11}(\theta=0), a_{2 k}^{1}=0, \forall k \geq 1 .
$$

Below, the analytic expressions for the first thirteen coefficients of the complete asymptotic M. Williams expansion, obtained with the expressions (16)-(19), are presented:

$$
\begin{gathered}
a_{1}^{1}=\frac{\sigma_{22}^{\infty}}{\sqrt{2 a}} \frac{a^{2}-c}{\sqrt{b^{2}-a^{2}}}, a_{2}^{1}=\frac{(\alpha-1)}{4} \sigma_{22}^{\infty}, \quad a_{2 k}^{1}=0, \forall k>1, \\
a_{3}^{1}=-\frac{\sigma_{22}^{\infty} \sqrt{2}}{24} \frac{3 a^{4}-7 a^{2} b^{2}+5 a^{2} c-b^{2} c}{a^{3 / 2}\left(b^{2}-a^{2}\right)^{3 / 2}}, \\
=-\frac{\sigma_{22}^{\infty} \sqrt{2}}{320} \frac{2 a^{2} b^{2} c-34 b^{2} a^{4}-19 b^{4} a^{2}+43 a^{4} c+5 a^{6}+3 a^{4} c}{a^{5 / 2}\left(b^{2}-a^{2}\right)^{5 / 2}} \\
a_{7}^{1}=-\frac{\sigma_{22}^{\infty} \sqrt{2}}{1792} \times \\
\times\left(-5 b^{6} c+137 b^{2} a^{4} c+11 b^{4} a^{2} c+177 a^{6} c+\right. \\
\left.+13 b^{6} a^{2}+7 a^{8}-113 b^{2} a^{6}-227 a^{4} b^{4}\right) /\left(a^{7 / 2}\left(b^{2}-a^{2}\right)^{7 / 2}\right)
\end{gathered}
$$

$$
\begin{gathered}
a_{9}^{1}=-\frac{\sigma_{22}^{\infty} \sqrt{2}}{36864} \frac{1}{a^{9 / 2}\left(b^{2}-a^{2}\right)^{9 / 2}} \times \\
\times\left\{45 a^{10}+5732 b^{2} a^{6} c+450 a^{4} b^{4} c+2867 a^{8} c+35 b^{8} c-\right. \\
\left.-124 b^{6} a^{2} c-67 b^{8} a^{2}-132 b^{6} a^{4}-1636 b^{2} a^{8}-7170 b^{4} a^{6}\right\} \\
\times\left\{10398 b^{4} a^{6} c+42997 b^{2} a^{8} c+77 a^{12}-10986 a^{6} b^{6}-63 b^{10} c-\right. \\
-367 b^{8} a^{4}-646 b^{6} a^{4} c+295 b^{8} a^{2} c+11531 a^{10} c- \\
\left.-6189 b^{2} a^{10}-47150 b^{4} a^{8}+103 b^{10} a^{2}\right\} \\
\left.-1764 a^{6} b^{6} c-3329 b^{8} a^{4} c+1330 b^{10} a^{2} c-231 b^{12} c\right\} \\
a_{13}^{1}=\frac{\sigma_{22}^{\infty} \sqrt{2}}{1703936} \frac{1}{a^{13 / 2}\left(b^{2}-a^{2}\right)^{13 / 2}} \times \\
\times\left\{48014 b^{2} a^{12}+559049 b^{4} a^{10}+329124 b^{6} a^{8}+11729 b^{8} a^{6}-\right. \\
-92479 a^{12} c-1810 b^{10} a^{4}-273 a^{14}+343 b^{12} a^{2}-
\end{gathered}
$$

Fig. 13 demonstrates the intensity level lines of the shearing strain intensity in the neighborhood of the tip $z=a$, built with the expansion (15), where the expansion coefficients are calculated with the formulae (16)-(19). Fig. 13 presents the results of a computation based on holding the main member of the stress tensor component asymptotic expansion (Fig. 13,a), and with the binomial asymptotic expansions (Fig. 13, b). The presented formulae mean that the second term, the T-stress, makes an impact on the component $\sigma_{11}^{1}$ and the stress intensity. Fig. 13, $b$ shows the level lines obtained through holding 15 terms. The further increase of the number of the terms held in the asymptotic expansion shows that there is no difference for the considered distances to the crack tip.

Table presents the numerical values of the stress intensity factors by the crack tip $z=a$ and $z=b$ for the plate medium with two collinear cracks. As we can see in Table, the stress intensity factors depend on the geometry of the plate medium with two cracks: on their lengths and distances between them. The farther from each other the cracks are in the plate, the weaker is their impact on each other. For the distances between the crack centers of the order $d>10 l$ each crack behaves as a separate crack and hardly interacts with the other crack in the plate medium. It is confirmed with the approximation of the numerical values of the stress intensity coefficients as the distances between the two cracks of the same length in the plate medium are increased. 


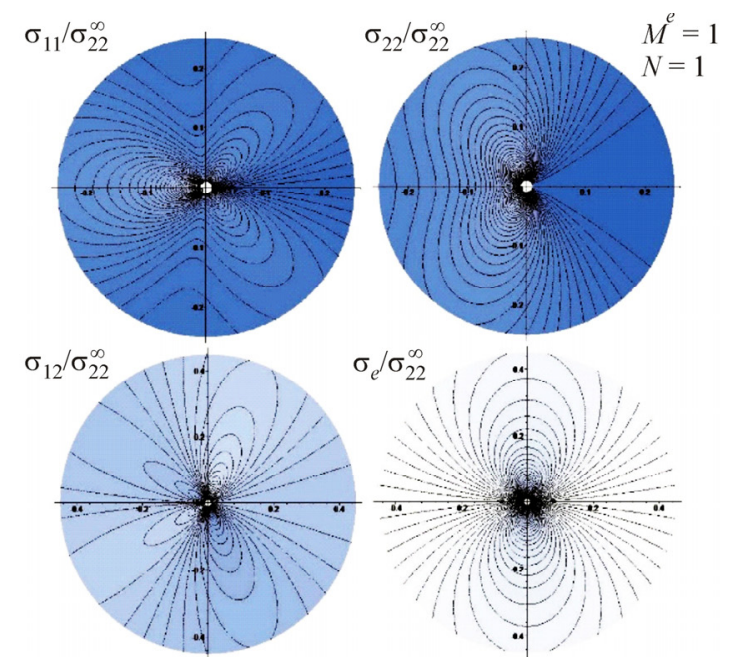

$a$
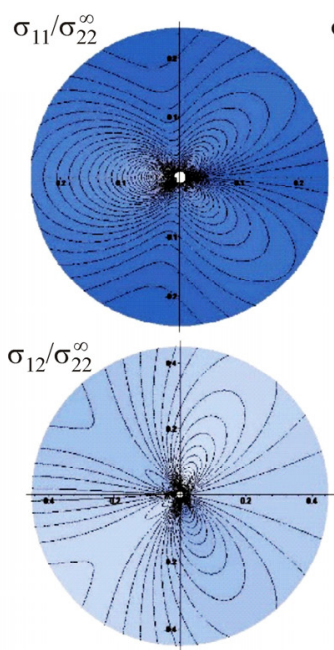

b
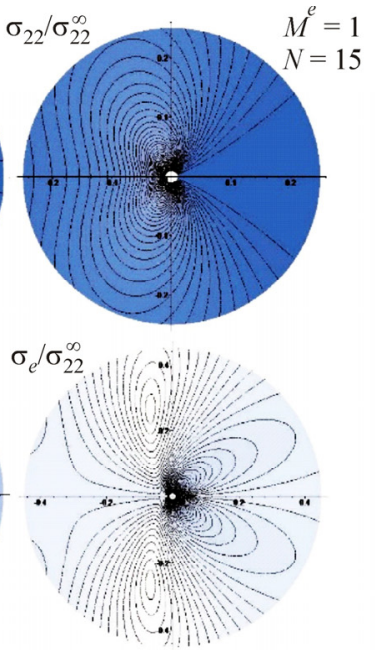

Fig. 13. The level lines of the stress tensor and stress intensity in the vicinity of the tip $z=a$, built with the mononomial $(a)$ and the fifteen-nomial asymptotic expansions $(b)$

Numerical values of the stress intensity factors for the plate medium with two collinear cracks

\begin{tabular}{|c|c|c|c|c|c|c|}
\hline \multicolumn{7}{|c|}{$\begin{array}{l}\text { Numerical values of the stress intensity factors for the plate medium with two collinear cracks of the length } 2 l \\
\text { and the distance between the crack middles } d\end{array}$} \\
\hline Crack half-length & $l, \mathrm{~cm}$ & 0.55 & 0.55 & 0.55 & 0.55 & 0.55 \\
\hline Distance between the crack middles & $d, \mathrm{~cm}$ & 1.11 & 1.65 & 2.75 & 3.85 & 5.5 \\
\hline Stress intensity factor by the tip $z=a$ & $K_{I}(a) / \sigma_{22}^{\infty}, \mathrm{cm}^{1 / 2}$ & 4.0823 & 1.4623 & 1.3502 & 1.3308 & 1.3219 \\
\hline Stress intensity factor by the tip $z=b$ & $K_{I}(\mathrm{~b}) / \sigma_{22}^{\infty}, \mathrm{cm}^{1 / 2}$ & 1.5886 & 1.3824 & 1.3380 & 1.3266 & 1.3206 \\
\hline Crack half-length & $l, \mathrm{~cm}$ & 0.6 & 0.6 & 0.6 & 0.6 & 0.6 \\
\hline Distance between the crack middles & $d, \mathrm{~cm}$ & 1.21 & 1.8 & 3 & 4.2 & 6 \\
\hline Stress intensity factor by the tip $z=a$ & $K_{I}(a) / \sigma_{22}^{\infty}, \mathrm{cm}^{1 / 2}$ & 4.3957 & 1.5274 & 1.4102 & 1.3899 & 1.3807 \\
\hline Stress intensity factor by the tip $z=b$ & $K_{I}(\mathrm{~b}) / \sigma_{22}^{\infty}, \mathrm{cm}^{1 / 2}$ & 1.6625 & 1.4439 & 1.3975 & 1.3856 & 1.3793 \\
\hline Crack half-length & $l, \mathrm{~cm}$ & 0.8 & 0.8 & 0.8 & 0.8 & 0.8 \\
\hline Distance between the crack middles & $d, \mathrm{~cm}$ & 1.61 & 2.4 & 4 & 4.8 & 8 \\
\hline Stress intensity factor by the tip $z=a$ & $K_{I}(a) / \sigma_{22}^{\infty}, \mathrm{cm}^{1 / 2}$ & 5.6205 & 1.7636 & 1.6284 & 1.6050 & 1.5943 \\
\hline Stress intensity factor by the tip $z=b$ & $K_{I}(\mathrm{~b}) / \sigma_{22}^{\infty}, \mathrm{cm}^{1 / 2}$ & 1.9318 & 1.6673 & 1.6137 & 1.6000 & 1.5927 \\
\hline Crack half-length & $l, \mathrm{~cm}$ & 1 & 1 & 1 & 1 & 1 \\
\hline Distance between the crack middles & $d, \mathrm{~cm}$ & 2.1 & 3 & 5 & 7 & 10 \\
\hline Stress intensity factor by the tip $z=a$ & $K_{I}(a) / \sigma_{22}^{\infty}, \mathrm{cm}^{1 / 2}$ & 3.1815 & 1.9718 & 1.8206 & 1.7944 & 1.7825 \\
\hline Stress intensity factor by the tip $z=b$ & $K_{I}(\mathrm{~b}) / \sigma_{22}^{\infty}, \mathrm{cm}^{1 / 2}$ & 2.0401 & 1.8641 & 1.8041 & 1.7888 & 1.7806 \\
\hline Crack half-length & $l, \mathrm{~cm}$ & 3 & 3 & 3 & 3 & 3 \\
\hline Distance between the crack middles & $d, \mathrm{~cm}$ & 6.1 & 9 & 15 & 21 & 30 \\
\hline Stress intensity factor by the tip $z=a$ & $K_{I}(a) / \sigma_{22}^{\infty}, \mathrm{cm}^{1 / 2}$ & 7.7557 & 3.4153 & 3.1534 & 3.1080 & 3.0874 \\
\hline Stress intensity factor by the tip $z=b$ & $K_{I}(\mathrm{~b}) / \sigma_{22}^{\infty}, \mathrm{cm}^{1 / 2}$ & 3.6544 & 3.2286 & 3.1248 & 3.0984 & 3.0842 \\
\hline Crack half-length & $l, \mathrm{~cm}$ & 5 & 5 & 5 & 5 & 5 \\
\hline Distance between the crack middles & $d, \mathrm{~cm}$ & 10.1 & 15 & 25 & 35 & 50 \\
\hline Stress intensity factor by the tip $z=a$ & $K_{I}(a) / \sigma_{22}^{\infty}, \mathrm{cm}^{1 / 2}$ & 11.9075 & 4.4091 & 4.0710 & 4.0124 & 3.9858 \\
\hline Stress intensity factor by the tip $z=b$ & $K_{I}(\mathrm{~b}) / \sigma_{22}^{\infty}, \mathrm{cm}^{1 / 2}$ & 4.7790 & 4.1682 & 4.0341 & 4.0000 & 3.9817 \\
\hline
\end{tabular}




\section{Analytic Determination of the Complete Asymptotic M. Williams Expansion Coefficients of the Stress Field for the Transverse Shear Crack Tip}

The asymptotic expansion of a complex potential (5) in the neighborhood of the crack tip $z(r, \theta)=b+r e^{i \theta}$, $x_{2}(r, \theta)=r \sin \theta$ has the form

$$
\varphi_{2}^{\prime}(z)=-i \frac{\sigma_{12}^{\infty}}{2} \sum_{n=0}^{\infty} q_{n}(z-b)^{n-1 / 2}+i \frac{\sigma_{12}^{\infty}}{2}
$$

where

$$
\begin{gathered}
q_{n}=\sum_{k=0}^{n+1} p_{k} d_{n-k}, \quad p_{n}=\sum_{k=0}^{n+1} c_{k} e_{n-k}, \quad e_{n}=\sum_{k=0}^{n+1} a_{k} b_{n-k}, \\
d_{0}=b^{2}-c, \quad d_{1}=2 b, \quad d_{2}=1, \quad d_{k}=0, k>2, \\
a_{k}=(-1)^{k} \frac{(|2 k-1| ! !)}{2^{k} k !}(b-a)^{-(2 k+1) / 2}, \\
b_{k}=(-1)^{k} \frac{(|2 k-1| ! !)}{2^{k} k !}(a+b)^{-(2 k+1) / 2}, \\
c_{k}=(-1)^{k} \frac{(|2 k-1| ! !)}{2^{k} k !}(2 b)^{-(2 k+1) / 2} .
\end{gathered}
$$

Formulae (21)-(24) contain the dependence of the asymptotic expansion coefficients of the complex potential on the geometric parameters of the specimen $a, b$ and the applied load $\sigma_{12}^{\infty}$. Substituting the expression (20) into the Kolosoff-Muskhelishvili formulae (5) and comparison with the complete asymptotic M. Williams expansion (1) leads to the determination of the amplitude coefficients of the expansion:

$$
a_{k}^{2}=\sigma_{12}^{\infty} q_{k} / f_{k}^{2,12}(\theta=0), \quad a_{2 k}^{1}=0, \forall k \geq 1 .
$$

Below, the analytic expressions for the first thirteen coefficients of the complete asymptotic M. Williams expansion, obtained with the expressions (21)-(24), are presented:

$$
\begin{gathered}
a_{1}^{2}=\frac{\sigma_{12}^{\infty}}{\sqrt{2 b}} \frac{b^{2}-c}{\sqrt{b^{2}-a^{2}}}, a_{2 k}^{2}=0, \forall k \geq 1, \\
a_{3}^{2}=\frac{\sigma_{12}^{\infty} \sqrt{2}}{24} \frac{3 b^{4}-7 a^{2} b^{2}+5 b^{2} c-a^{2} c}{b^{3 / 2}\left(b^{2}-a^{2}\right)^{3 / 2}}, \\
a_{5}^{2}=-\frac{\sigma_{12}^{\infty} \sqrt{2}}{320} \times \\
\frac{2 a^{2} b^{2} c-34 a^{2} b^{4}-19 a^{4} b^{2}+43 b^{4} c+5 b^{6}+3 a^{4} c}{b^{5 / 2}\left(b^{2}-a^{2}\right)^{5 / 2}}, \\
a_{7}^{2}=\frac{\sigma_{12}^{\infty} \sqrt{2}}{1792} \times
\end{gathered}
$$

$$
\begin{gathered}
\times\left(-5 a^{6} c+137 a^{2} b^{4} c+11 a^{4} b^{2} c+177 b^{6} c+\right. \\
\left.+13 a^{6} b^{2}+7 b^{8}-113 a^{2} b^{6}-227 a^{4} b^{4}\right) /\left(b^{7 / 2}\left(b^{2}-a^{2}\right)^{7 / 2}\right) \\
a_{9}^{2}=-\frac{\sigma_{12}^{\infty} \sqrt{2}}{36864} \frac{1}{b^{9 / 2}\left(b^{2}-a^{2}\right)^{9 / 2}} \times \\
\times\left\{45 b^{10}+5732 a^{2} b^{6} c+450 a^{4} b^{4} c+2867 b^{8} c+35 a^{8} c-\right. \\
\left.-124 a^{6} b^{2} c-67 a^{8} b^{2}-132 a^{6} b^{4}-1636 a^{2} b^{8}-7170 a^{4} b^{6}\right\} \\
a_{11}^{2}=\frac{\sigma_{12}^{\infty} \sqrt{2}}{180224} \frac{1}{b^{11 / 2}\left(b^{2}-a^{2}\right)^{11 / 2}} \times \\
\times\left\{10398 a^{4} b^{6} c+42997 a^{2} b^{8} c+77 b^{12}-10986 a^{6} b^{6}-\right. \\
-367 a^{8} b^{4}-646 a^{6} b^{4} c+295 a^{8} b^{2} c+11531 b^{10} c- \\
\left.-6189 a^{2} b^{10}-47150 a^{4} b^{8}-63 a^{10} c+103 a^{10} b^{2}\right\}
\end{gathered}
$$

Substituting the asymptotic presentation for the complex potential (20) into (4), it is possible to acquire the asymptotic presentations of the stress tensor components in vicinity of the crack tip $z=b$ under the transverse shear conditions

$$
\sigma_{i j}^{2}(r, \theta)=\sigma_{12}^{\infty} \sum_{n=0}^{\infty} q_{n} g_{n}^{2, i j}(\theta) r^{n-1 / 2}
$$

where the angular distributions $g_{n}^{m, i j}(\theta)$ are determined as

$$
g_{n}^{2,11}(\theta)=
$$

$=[(n+7 / 2) \sin (n-1 / 2) \theta-(n-1 / 2) \sin (n-5 / 2) \theta] / 2$,

$$
g_{n}^{2,22}(\theta)=
$$

$$
\begin{gathered}
=[(-n+1 / 2) \sin (n-1 / 2) \theta+(n-1 / 2) \sin (n-5 / 2) \theta] / 2, \\
g_{n}^{2,12}(\theta)=
\end{gathered}
$$$$
=[(n+3 / 2) \cos (n-1 / 2) \theta-(n-1 / 2) \cos (n-5 / 2) \theta] / 2 \text {. }
$$

Fig. 14 shows the level lines of the stress tensor components and the stress intensity in the vicinity of the tip $z=b$, built with the mononomial $(a)$ and the thirty-fivenomial asymptotic expansions $(b)$. Fig. 15 presents the angular shearing strain distributions $\sigma_{12}^{2}$ in the vicinity of the crack tip $z=b$ in different distances from the crack tip. It is found that in the close vicinity from the crack tip it is quite hard to hold the three terms in the neighborhood of the crack tip (Fig. 15, a) while as the distance increases, the trinomial, five-nomial and seven-nomial asymptotic expansions become distinguishable and the higher approximations need to be held. 


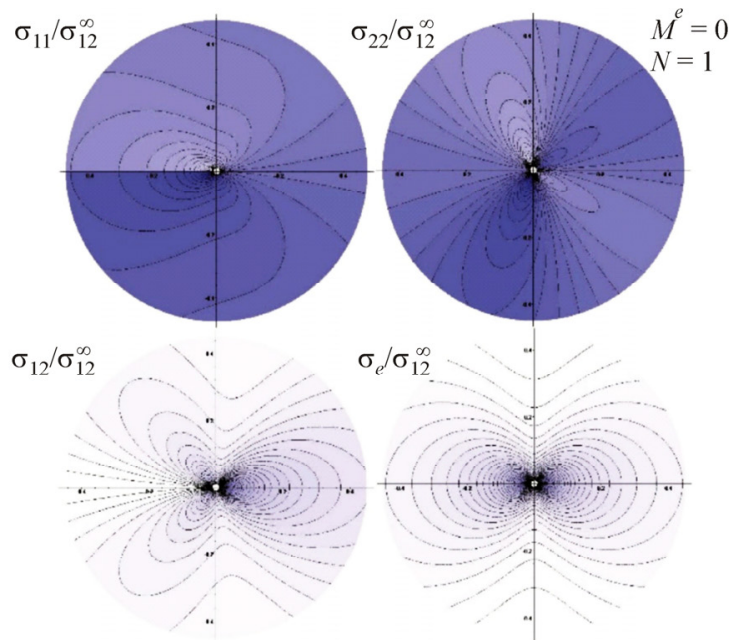

$a$
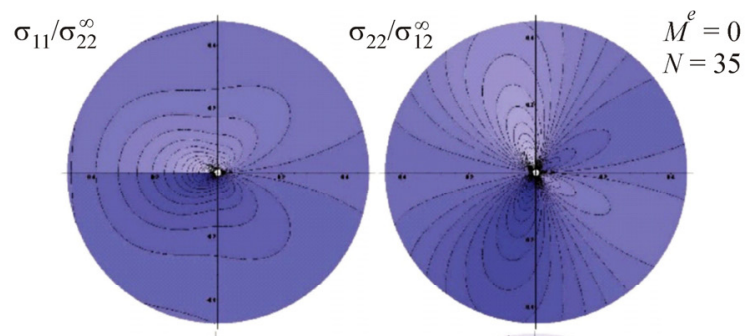

$\sigma_{12} / \sigma_{12}^{\infty}$

$\sigma_{e} / \sigma_{12}^{\infty}$

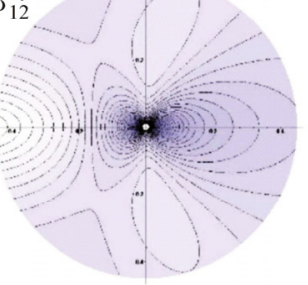

Fig. 14. The stress tensor component expansion near the crack tip $z=b$ for the main member of the asymptotic expansion $(a)$ and for the expansion of 35 terms $(b)$
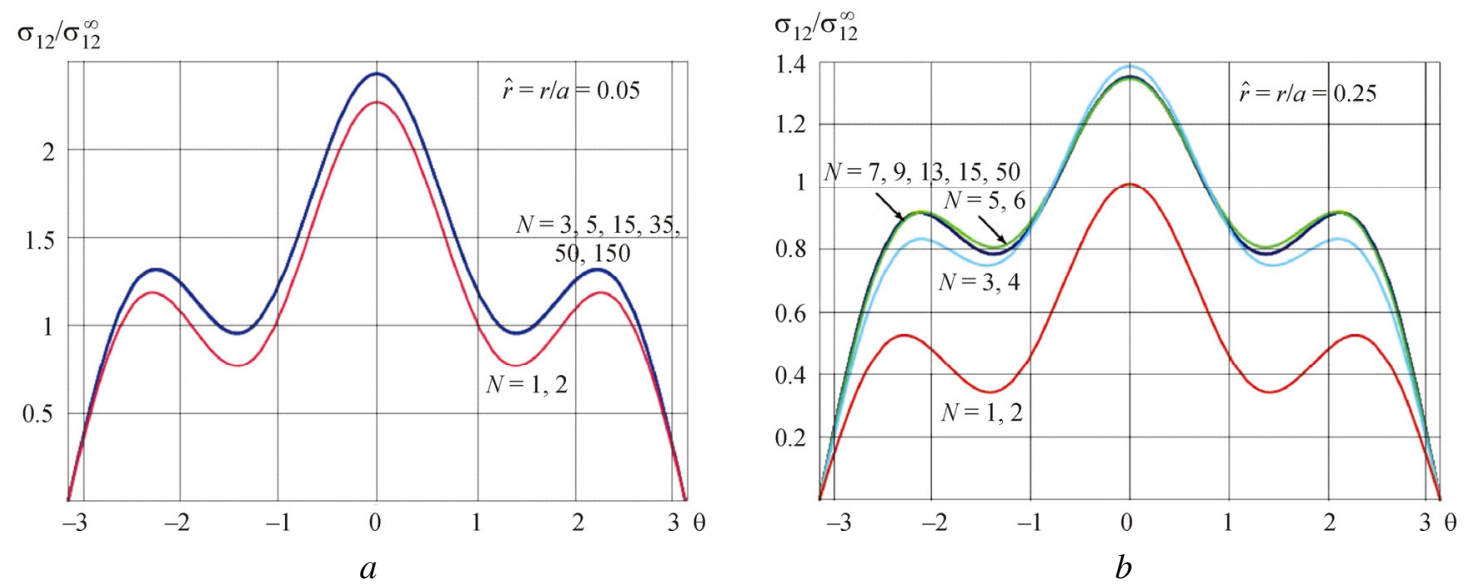

Fig. 15. Angular shearing strain distributions $\sigma_{12}^{2}$ in the vicinity of the crack tip $z=b: a$ ) for $\left.\hat{r}=r / a=0.05 ; b\right)$ for $\hat{r}=r / a=0.25$
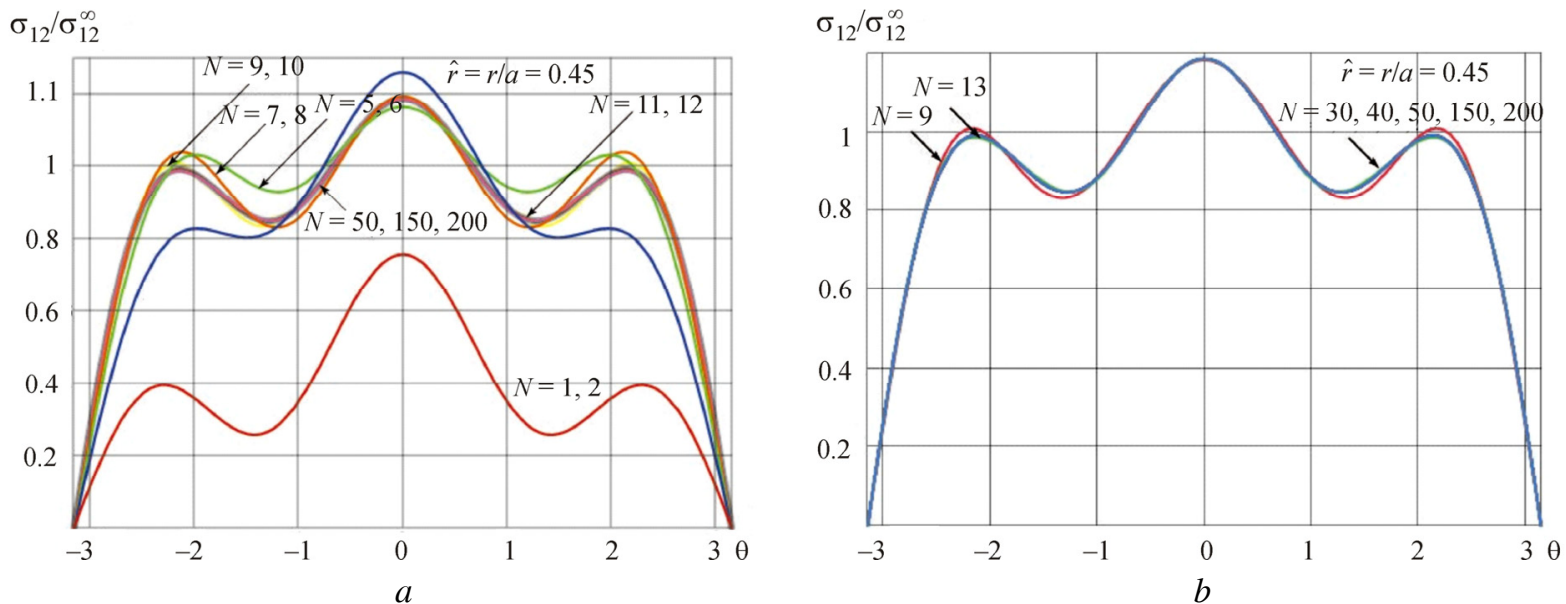

Fig. 16. Angular stress distributions $\sigma_{12}^{2}$ in the neighborhood of the crack tip $z=b$, built with the multiparameter asymptotic expansion with holding various numbers of terms in the asymptotic expansion

Fig. 16 presents the angular shearing strain $\sigma_{12}^{2}$ distributions in the vicinity of the crack tip $z=b$ for $\hat{r}=r / a=0.45$. In Fig. 16 it is seen that the nine-nomial and thirteen-nomial asymptotic expansions are different from each other. Fig. 17 presents the angular distributions of the stress tensor component $\sigma_{12}^{2}$ in the vicinity of the crack tip $z=b$ for $\hat{r}=r / a=0.75$.

From the presented distributions of the stress tensor component $\sigma_{12}^{2}$ we can see, that the expansion of the fairness scope of $\mathrm{M}$. Williams asymptotics requires 

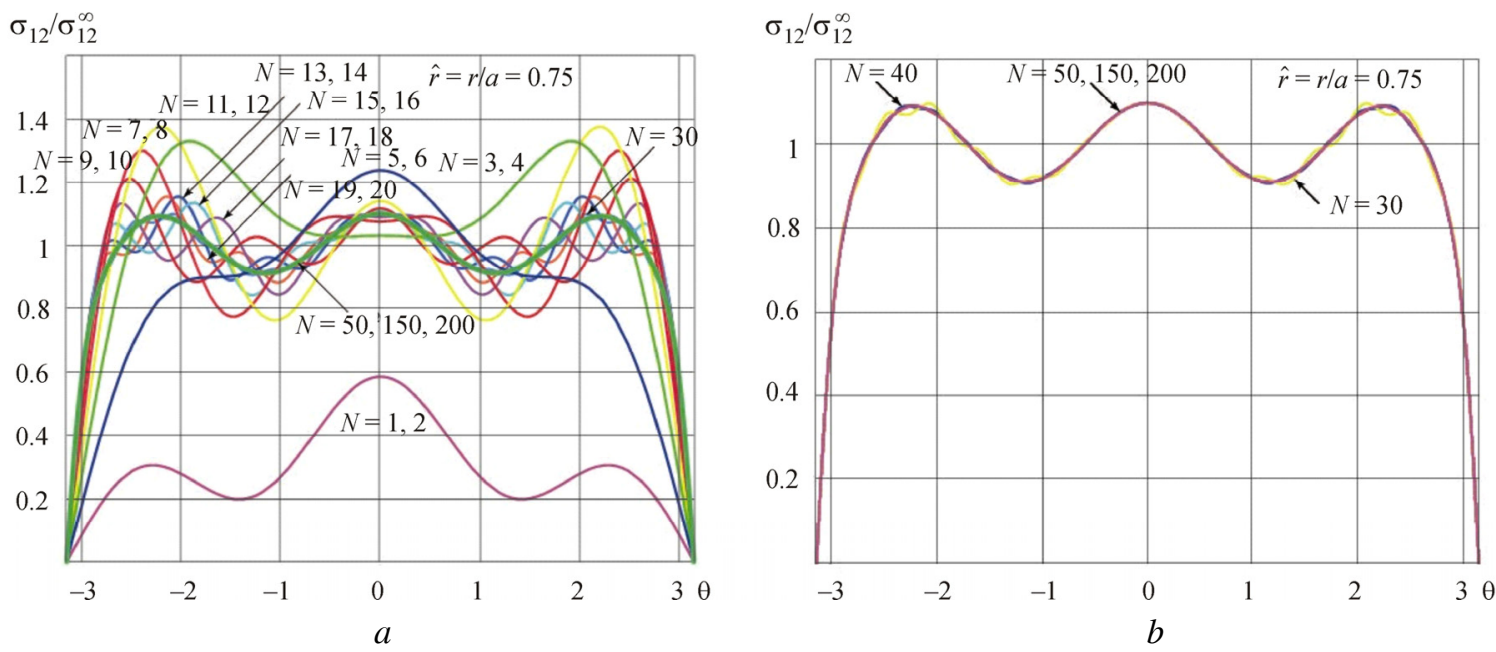

Fig. 17. Angular stress distributions $\sigma_{12}^{2}$ in the neighborhood of the crack tip $z=b$, built with the multiparameter asymptotic expansion with holding various numbers of terms in the asymptotic expansion

holding the higher approximations up to 40 terms. After 40 terms, the angular distributions are not distinguishable anymore: Fig. 17, $b$ shows the angular distributions built with the asymptotic expansions containing 30, 40, 50, 150 and 200 terms. The angular distribution built with 30 terms in an asymptotic expansion, is different from the other curves, as after holding 40 terms the curves are not distinguishable anymore (unlike Fig. 15, $a$ built for $\hat{r}=r / a=0.05$, where three terms of the asymptotic expansion are enough).

The asymptotic expansion of a complex potential (5) in the neighborhood of the crack tip $z(r, \theta)=a+r e^{i \theta}$, $x_{2}(r, \theta)=r \sin \theta$ has the form

$$
\varphi_{2}{ }^{\prime}(z)=-i \frac{\sigma_{12}^{\infty}}{2} \sum_{n=0}^{\infty} \delta_{n}(z-a)^{n-1 / 2}+i \frac{\sigma_{12}^{\infty}}{2},
$$

where

$$
\begin{gathered}
\delta_{n}=\sum_{k=0}^{n+1} p_{k} d_{n-k}, \quad \chi_{n}=\sum_{k=0}^{n+1} \beta_{k} \xi_{n-k}, \quad \xi_{n}=\sum_{k=0}^{n+1} \varsigma_{k} \eta_{n-k}, \\
\eta_{0}=a^{2}-c, \quad \eta_{1}=2 b, \quad \eta_{2}=1, \quad \eta_{k}=0, k>2, \\
\alpha_{k}=(-1)^{k} \frac{(|2 k-1| ! !)}{2^{k} k !}(2 a)^{-(2 k+1) / 2}, \\
\beta_{k}=(-1)^{k} \frac{(|2 k-1| ! !)}{2^{k} k !}(b-a)^{-(2 k+1) / 2}, \\
\varsigma_{k}=(-1)^{k} \frac{(|2 k-1| ! !)}{2^{k} k !}(a+b)^{-(2 k+1) / 2} .
\end{gathered}
$$

Formulae (26)-(30) contain the dependence of the asymptotic expansion coefficients of the complex potential on the geometric parameters of the specimen $a, b$ and the applied load $\sigma_{12}^{\infty}$. Substituting the expression (26) into the Kolosoff-Muskhelishvili formulae (5) and comparison with the complete asymptotic M. Williams expansion (1) leads to the determination of the amplitude coefficients of the expansion:

$$
a_{k}^{2}=\sigma_{12}^{\infty} q_{k} / f_{k}^{2,12}(\theta=0), \quad a_{2 k}^{1}=0, \forall k \geq 1 .
$$

Below, the analytic expressions for the first thirteen coefficients of the complete asymptotic M. Williams expansion, obtained with the expressions (26)-(30), are presented:

$$
\begin{gathered}
a_{1}^{2}=-\frac{\sigma_{12}^{\infty}}{\sqrt{2 b}} \frac{a^{2}-c}{\sqrt{b^{2}-a^{2}}}, a_{2 k}^{2}=0, \forall k \geq 1, \\
a_{3}^{2}=\frac{\sigma_{12}^{\infty} \sqrt{2}}{24} \frac{3 a^{4}-7 a^{2} b^{2}+5 a^{2} c-a^{2} c}{a^{3 / 2}\left(b^{2}-a^{2}\right)^{3 / 2}}, \\
\times \frac{a_{5}^{2}=\frac{\sigma_{12}^{\infty} \sqrt{2}}{320} \times}{a^{2} b^{2} c-34 b^{2} a^{4}-19 b^{4} a^{2}+43 a^{4} c+5 a^{6}+3 b^{4} c} \\
\left.\times 124 b^{6} a^{2} c-67 b^{8} a^{2}-132 b^{6} a^{4}-1636 b^{2} a^{8}-7170 b^{4} a^{6}\right\}(31) \\
\left.\times 13 b^{6} a^{2}+7 a^{8}-113 b^{2} a^{6}-227 b^{4} a^{4}\right) /\left(a^{7 / 2}\left(b^{2}-a^{2}\right)^{7 / 2}\right. \\
\times\left\{4 a^{6} c+137 b^{2} a^{4} c+11 b^{4} a^{2} c+177 a^{6} c+\right. \\
a_{11}^{2}=\frac{\sigma_{12}^{\infty} \sqrt{2}}{180224} \frac{\sigma_{12}^{\infty} \sqrt{2}}{a^{11 / 2}\left(b^{2}-a^{2}\right)^{11 / 2}} \times \\
a_{9}^{2}=\frac{\sigma_{12}^{\infty} \sqrt{2}}{36864} \frac{1}{a^{9 / 2}\left(b^{2}-a^{2}\right)^{9 / 2}} \times
\end{gathered}
$$




$$
\begin{gathered}
\times\left\{10398 b^{4} a^{6} c+42997 b^{2} a^{8} c+77 a^{12}-10986 b^{6} a^{6}-\right. \\
-367 b^{8} a^{4}-646 b^{6} a^{4} c+295 b^{8} a^{2} c+11531 a^{10} c- \\
\left.-6189 b^{2} a^{10}-47150 b^{4} a^{8}-63 b^{10} c+103 b^{10} a^{2}\right\}, \\
a_{13}^{2}=-\frac{\sigma_{12}^{\infty} \sqrt{2}}{1703936} \frac{1}{a^{13 / 2}\left(b^{2}-a^{2}\right)^{13 / 2}} \times \\
\times\left\{48014 b^{2} a^{12}+559049 b^{4} a^{10}+329124 b^{6} a^{8}+11729 b^{8} a^{6}-\right. \\
-92479 a^{12} c-1810 b^{10} a^{4}-273 a^{14}+343 b^{12} a^{2}- \\
-551534 b^{2} a^{10} c-298169 b^{4} a^{8} c-1764 b^{6} a^{6} c- \\
\left.-3329 b^{8} a^{4} c+1330 b^{10} a^{2} c-231 b^{12} c\right\} .
\end{gathered}
$$

With the calculated coefficients (31) it is possible to build the stress tensor components distribution by the crack tip $z=a$, holding various numbers of terms in the asymptotic expansion. Fig. 18, $a$ shows level lines of the stress tensor components and the stress intensities obtained with a mononomial asymptotic expansion. Fig. 18, $b$ demonstrates the same values based on the asymptotic expansions with 15 terms. Obviously, the level line picture materially changes, especially for the stress intensity.

\section{Analytic Determination of the Complete Asymptotic M. Williams Expansion Coefficients of the Stress Field for the Mixed Loading Conditions}

Due to the linear nature of the linear elasticity theory problem, the principle of solution superposition (formula (1)) is right, and with the help of the asymptotic expansions built for the normal fracture cracks and the transverse shear, it is easy to find the stress distributions near the crack tip under mixed mode loading conditions within the full diapason of the mixed deformation forms, setting the loading promiscuity parameter values.

Fig. 19-21 show the level lines of the stress tensor components and the stress intensities in the direct vicinity of the tip $z=a$ for various forms of mixed deformation and various numbers of terms held in the asymptotic expansion. The presented figures demonstrate that the higher approximations in the asymptotic expansions make a visible impact on the stress tensor components distribution in the vicinity of the crack tip. Fig. 19 shows the results of the stress tensor components and stress intensity calculations for the promiscuity parameter $M^{e}=0.75$ for the mononomial (Fig. 19, a) and the binomial (Fig. 19, b) asymptotic stress expansions. The comparison of the figures show that the consideration of higher approximations makes a critical impact on the level lines (Fig. 20, $b$ ).

Fig. 20 presents the polar diagrams of the stress tensor components in the vicinity of the crack tip $z=a$ for the loading promiscuity parameter $M^{e}=0.5$. The conducted analysis and the built diagrams reveal, that considerations of the T-stresses alone do not make a significant impact on the distribution nature of the stress tensor components, making minor changes in the stress intensity. While consideration of the higher approximations is more prominent (Fig. 20, $b$ ).

Fig. 21 shows the level lines of the stress tensor components and the shearing strain intensity in the vicinity of the crack $z=a$ for $M^{e}=0.25$. For constructing the curves in Fig. 21, $a$, the mononomial asymptotic expansions of the stress tensor components were used. The analysis

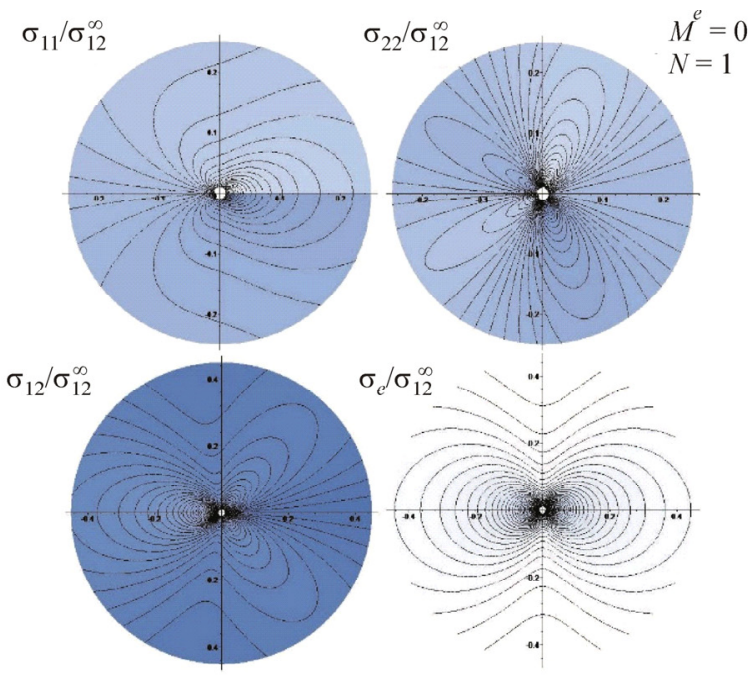

$a$

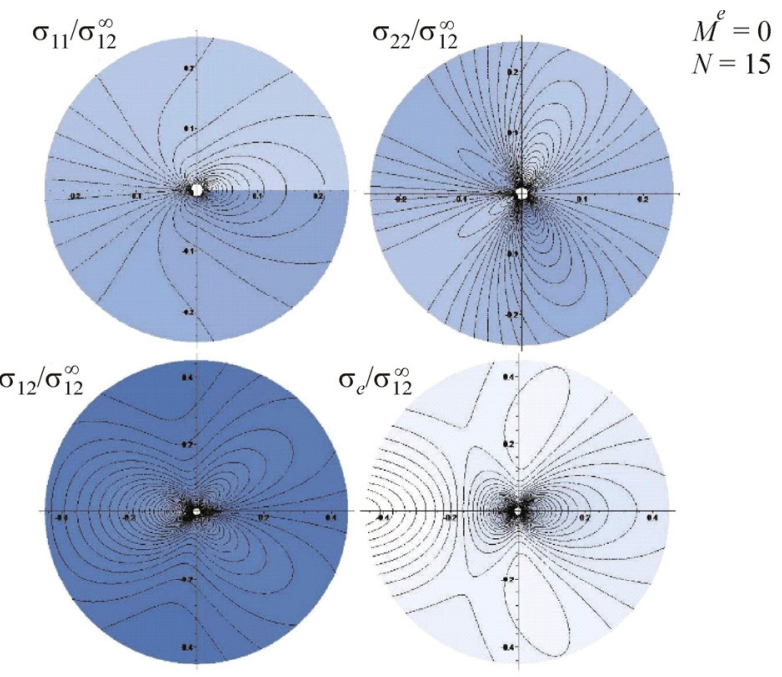

$b$

Fig. 18. The lines of equal values of the stress tensor component in the vicinity of the crack tip $z=a$ for a transverse shear crack, built with a mononomial $(a)$ and fifteen-nomial asymptotic expansion $(b)$ 


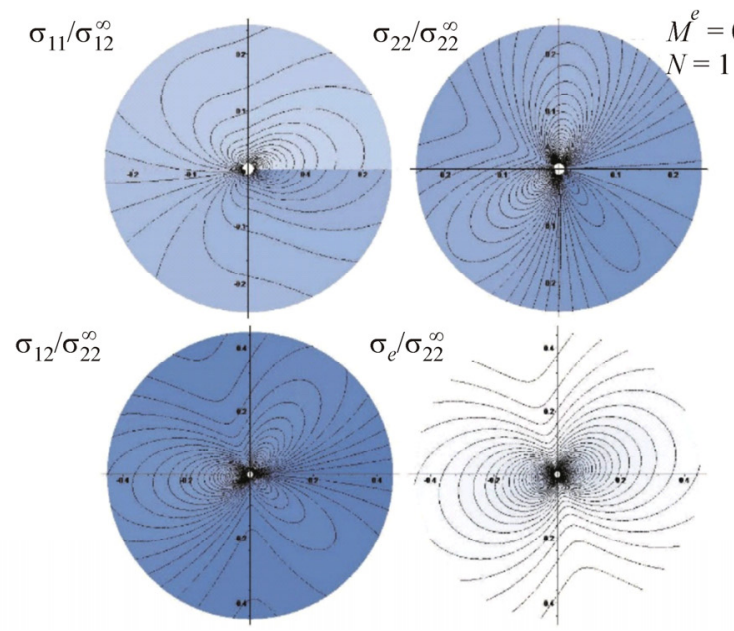

$a$
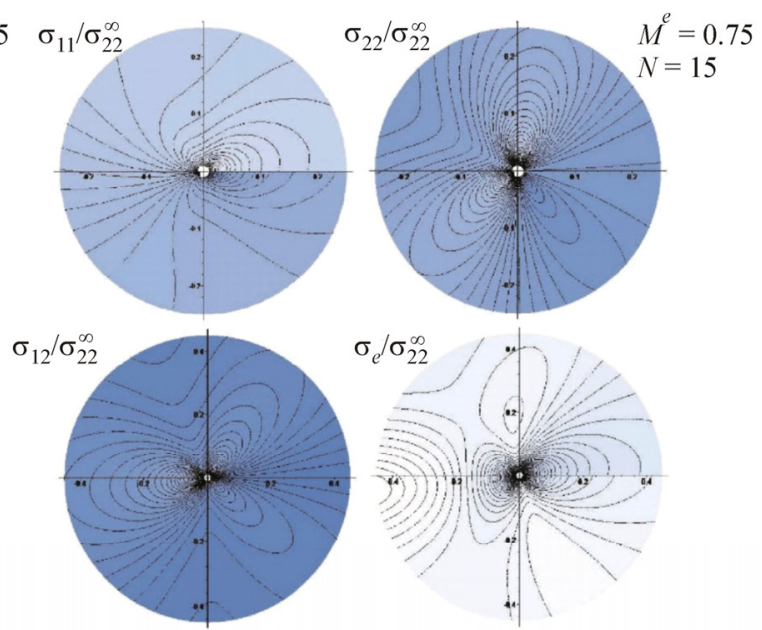

$\sigma_{e} / \sigma_{22}^{\infty}$

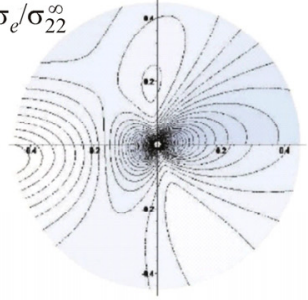

$b$

Fig. 19. Level lines of the stress tensor components in the vicinity of the crack tip $z=a$ I $\mathrm{n}$ the case of mixed mode loading, built with mononomial asymptotic expansions $(a)$ and by means of fifteen-nomial asymptotic expansions $(b)$

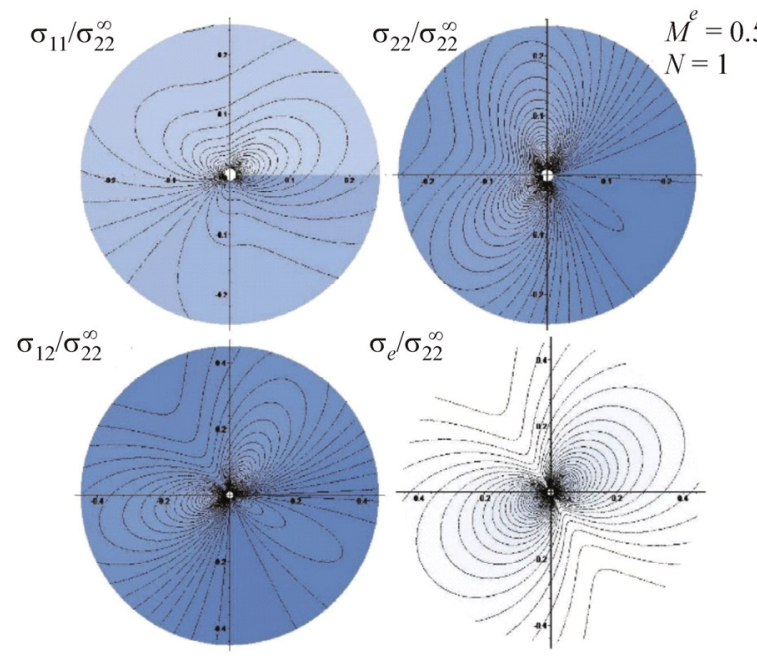

$a$

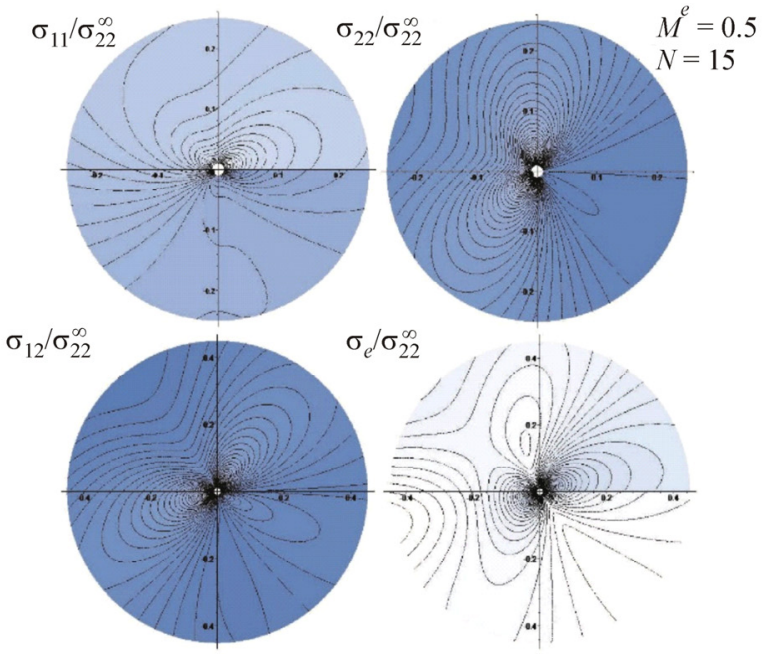

$b$

Fig. 20. The level lines of the stress tensor components in the vicinity of the crack tip $z=a$ in case of mixed mode loading conditions for different values of the loading promiscuity parameter: $M^{e}=0.5$, built with the mononomial asymptotic expansions $(a)$ and by means of the fifteen-term asymptotic expansions $(b)$

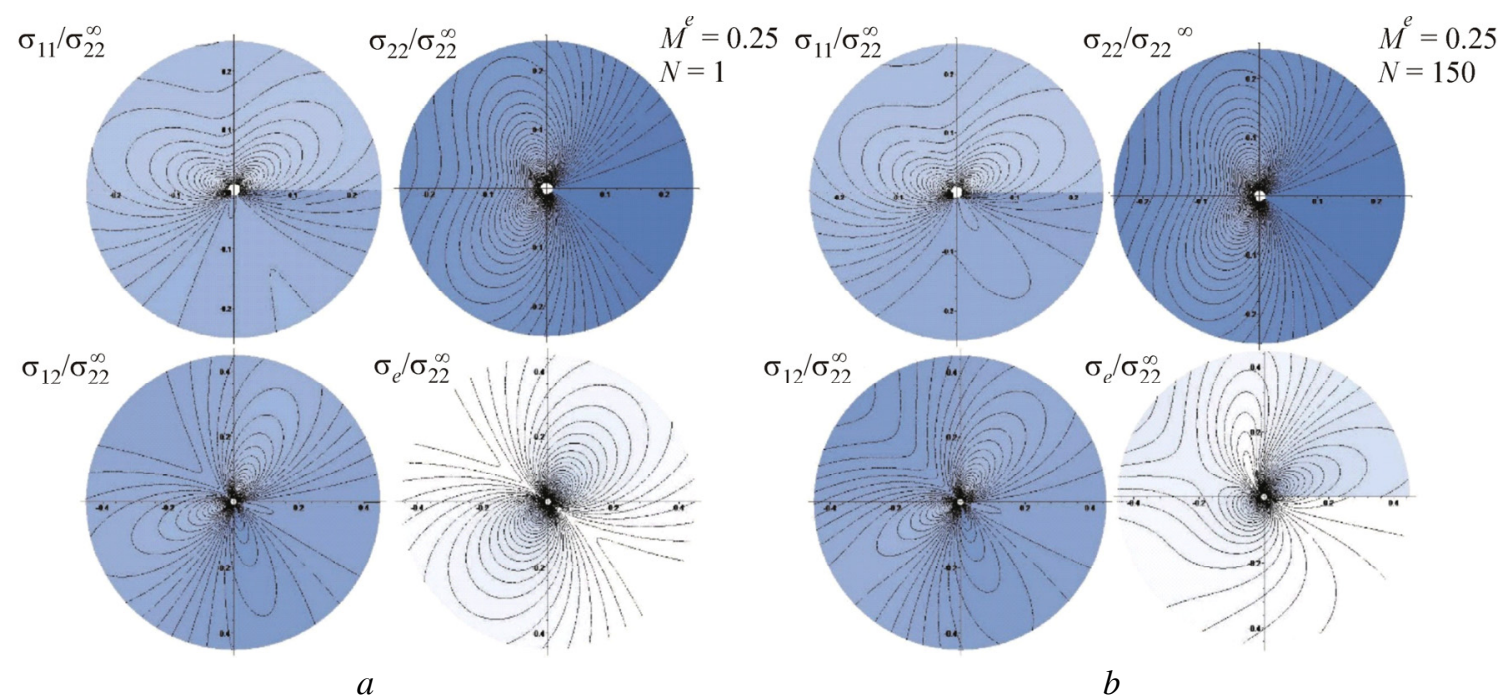

Fig. 21. The level lines of stress in the vicinity of the tip $z=a$ for the mononomial and binomial asymptotic expansions for the loading promiscuity parameter $M^{e}=0.25$, built with the mononomial asymptotic expansions $(a)$ and by means of the asymptotic expansions holding 150 terms $(b)$ 
revealed that holding the T-stresses only helps clarifying the stress intensity. Building the level lines in Fig. 21, b, 150 terms were held in the asymptotic expansion. It is seen that consideration of the higher approximations increases the calculation precision of the mechanic values of the crack tip. Fig. 22-25 demonstrate the angular distributions of the component $\sigma_{22}$ in the vicinity of the crack tip under mixed mode loading conditions applied in different distances from the crack tip. The figures show that the expansion of the asymptotic scope determined the need for holding the higher-order terms.
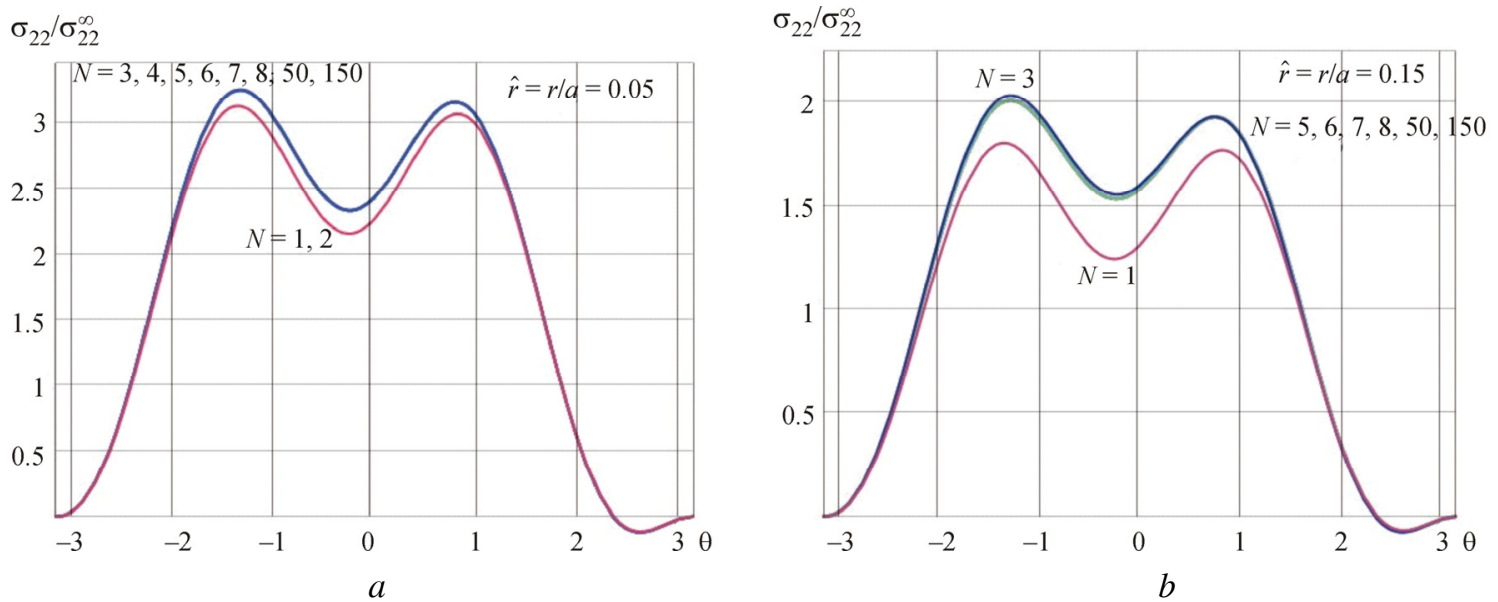

Fig. 22. The angular distribution of the stress tensor component $\sigma_{22}$ under mixed mode loading conditions $\left(\sigma_{11}^{\infty}, \sigma_{12}^{\infty}, \sigma_{22}^{\infty}\right)=(0,0.75,1) \sigma_{22}^{\infty}$ in different distances from the crack tip: $a$ ) for $\hat{r}=r / a=0.05 ; b)$ for $\hat{r}=r / a=0.15$
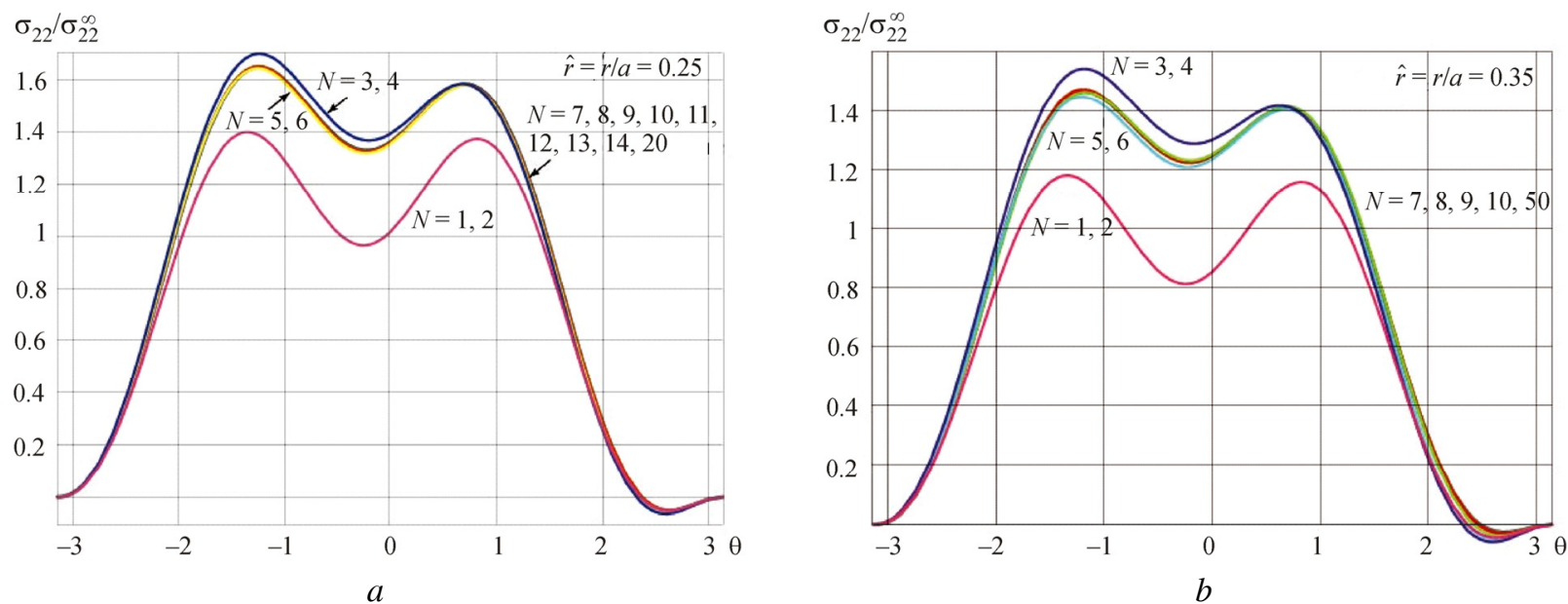

Fig. 23. The angular distribution of the stress tensor component $\sigma_{22}$ under mixed mode loading conditions $\left(\sigma_{11}^{\infty}, \sigma_{12}^{\infty}, \sigma_{22}^{\infty}\right)=(0,0.75,1) \sigma_{22}^{\infty}$ in different distances from the crack tip: $a$ ) for $\hat{r}=r / a=0.25 ; b)$ for $\hat{r}=r / a=0.35$
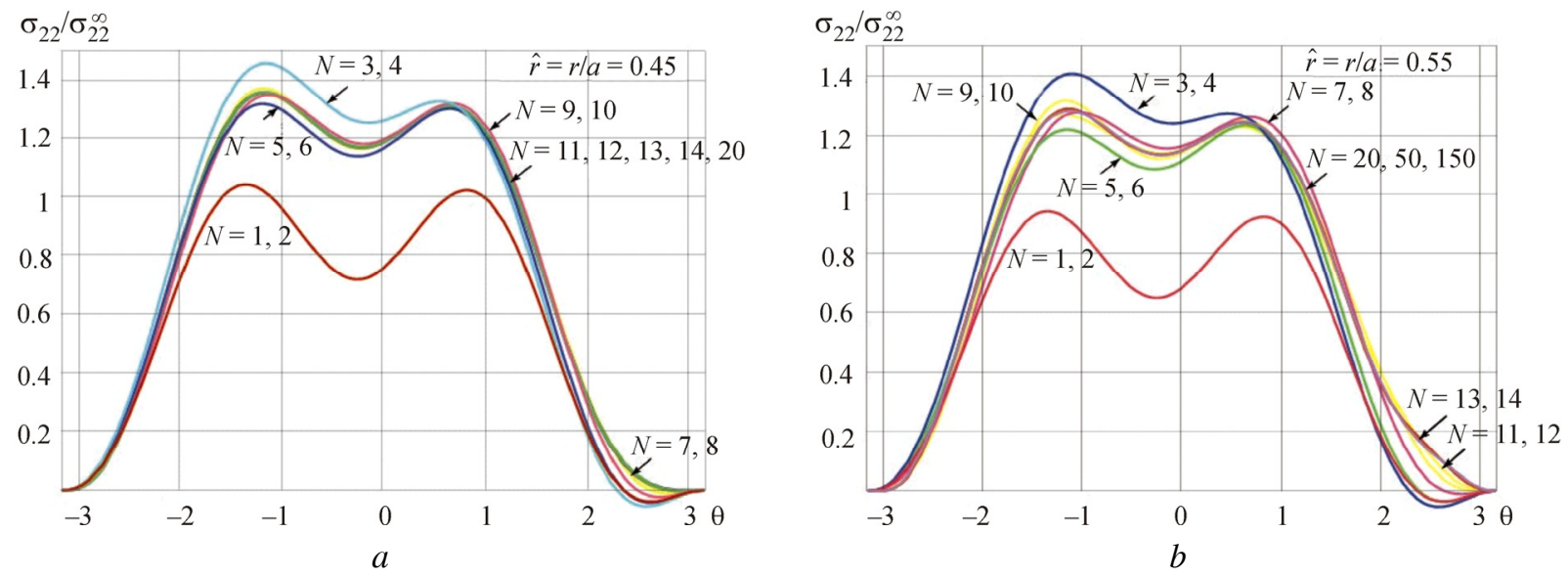

Fig. 24. The angular distribution of the stress tensor component $\sigma_{22}$ under mixed mode loading conditions $\left(\sigma_{11}^{\infty}, \sigma_{12}^{\infty}, \sigma_{22}^{\infty}\right)=(0,0.75,1) \sigma_{22}^{\infty}$ in different distances from the crack tip: $a$ ) for $\hat{r}=r / a=0.45 ; b)$ for $\hat{r}=r / a=0.55$ 


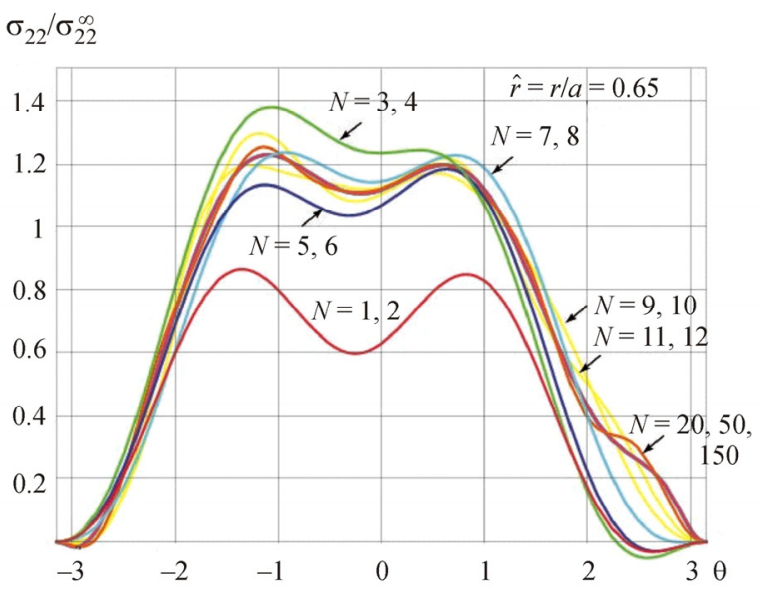

$a$

$$
\sigma_{22} / \sigma_{22}^{\infty}
$$

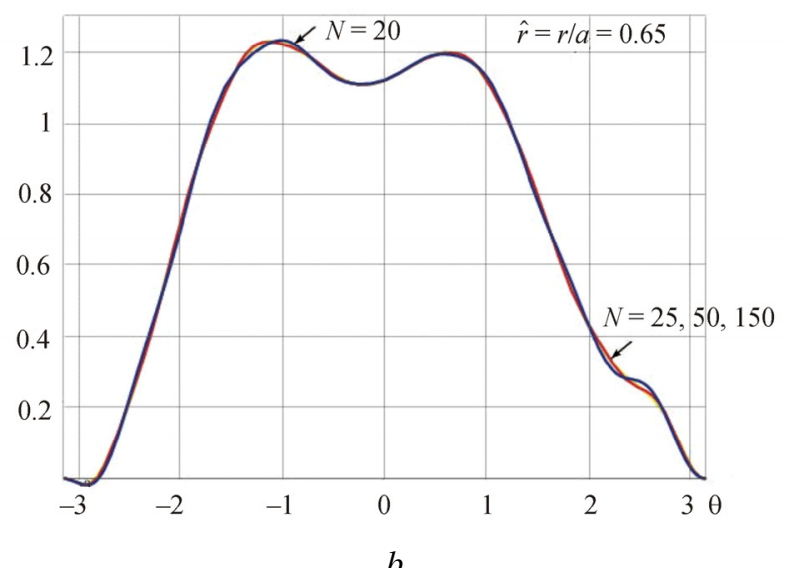

$b$

Fig. 25. The angular distributions of the stress tensor component $\sigma_{22}$ under mixed mode loading conditions $\left(\sigma_{11}^{\infty}, \sigma_{12}^{\infty}, \sigma_{22}^{\infty}\right)=(0,0.75,1) \sigma_{22}^{\infty}$, built with different numbers of held terms in asymptotic expansion
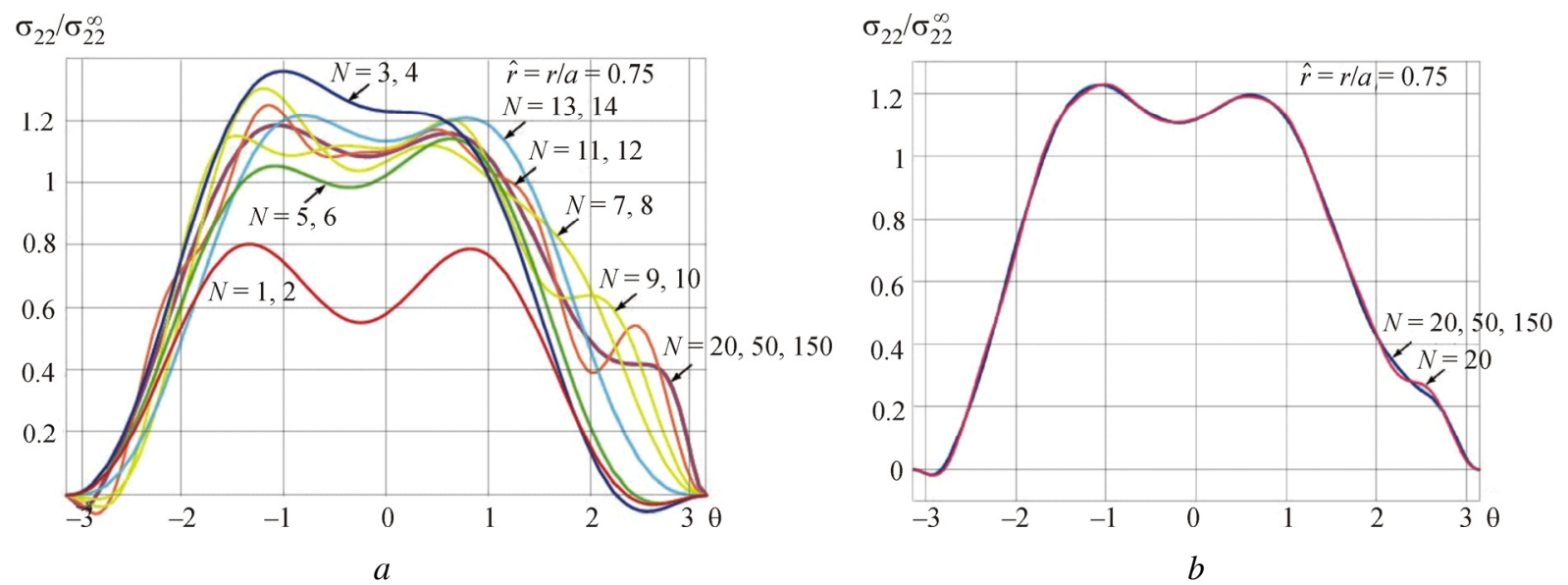

Fig. 26. The angular distributions of the stress tensor component $\sigma_{22}$ under mixed mode loading conditions $\left(\sigma_{11}^{\infty}, \sigma_{12}^{\infty}, \sigma_{22}^{\infty}\right)=(0,0.75,1) \sigma_{22}^{\infty}$, built with different numbers of held terms in asymptotic expansion
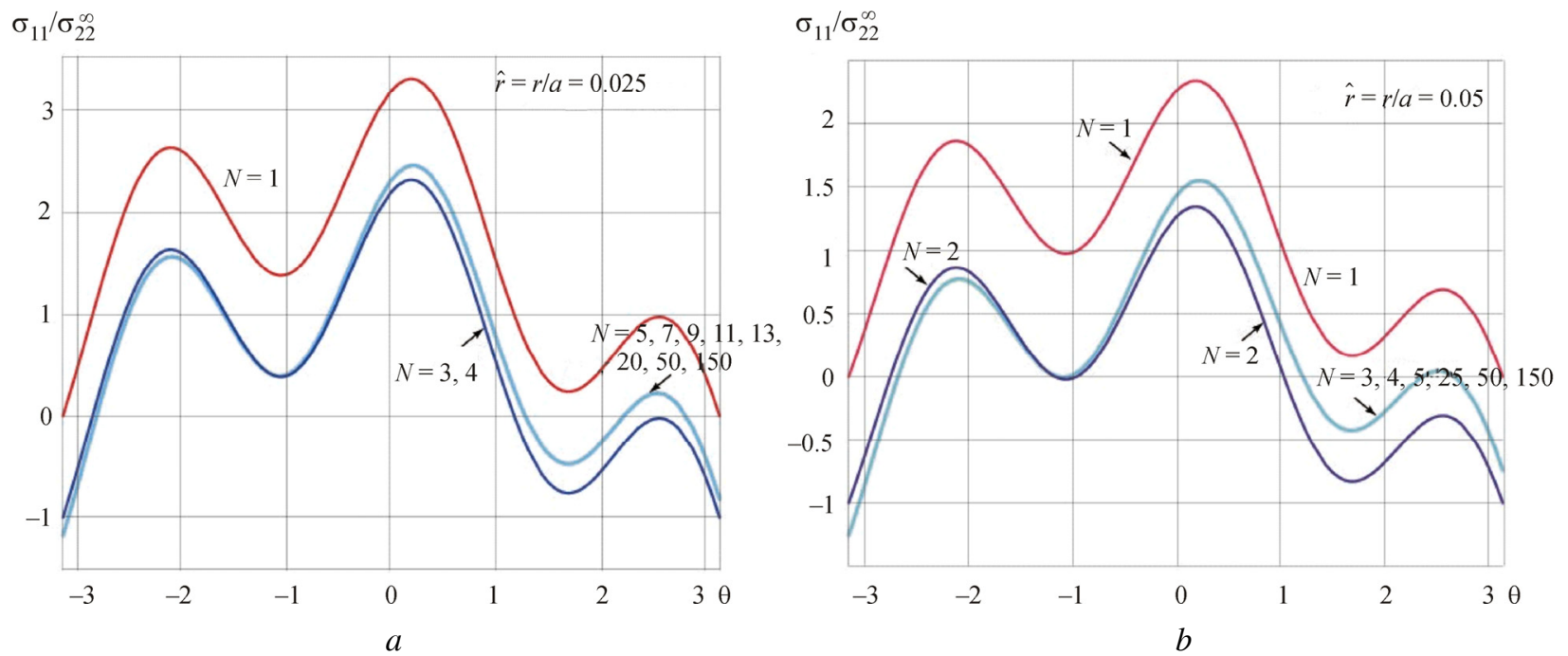

Fig. 27. The angular distribution of the stress tensor component $\sigma_{11}$ under mixed mode loading conditions $\left(\sigma_{11}^{\infty}, \sigma_{12}^{\infty}, \sigma_{22}^{\infty}\right)=(0,0.75,1) \sigma_{22}^{\infty}$ in different distances from the crack tip: $a$ ) for $\hat{r}=r / a=0.025 ; b)$ for $\hat{r}=r / a=0.05$ 

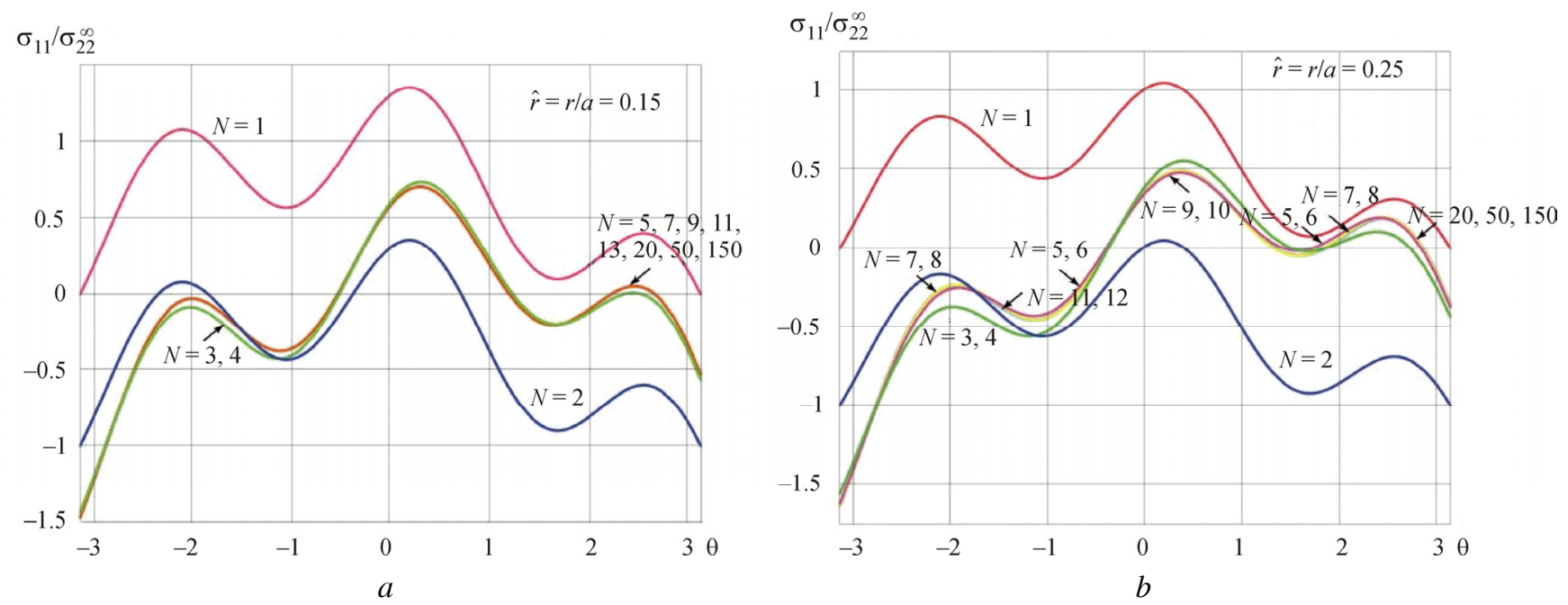

Fig. 28. The angular distribution of the stress tensor component $\sigma_{11}$ under mixed mode loading conditions $\left(\sigma_{11}^{\infty}, \sigma_{12}^{\infty}, \sigma_{22}^{\infty}\right)=(0,0.75,1) \sigma_{22}^{\infty}$ in different distances from the crack tip: $a$ ) for $\hat{r}=r / a=0.15 ; b)$ for $\hat{r}=r / a=0.25$

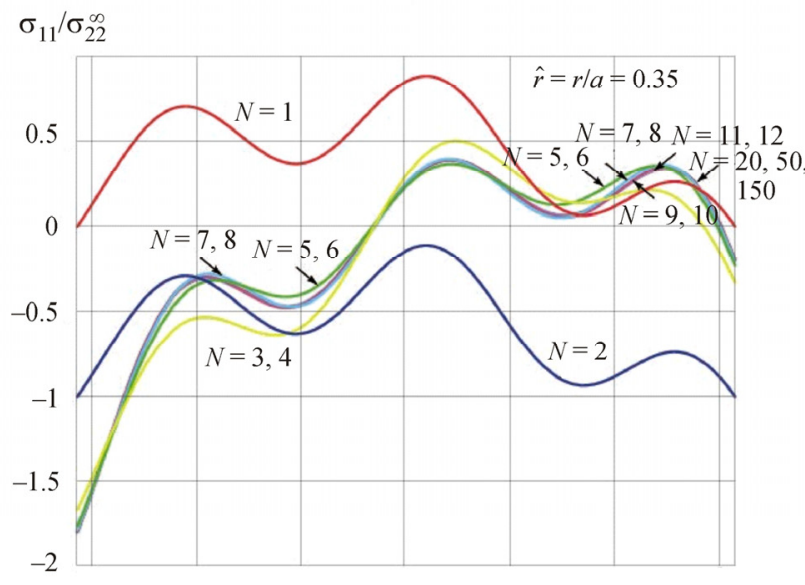

$a$

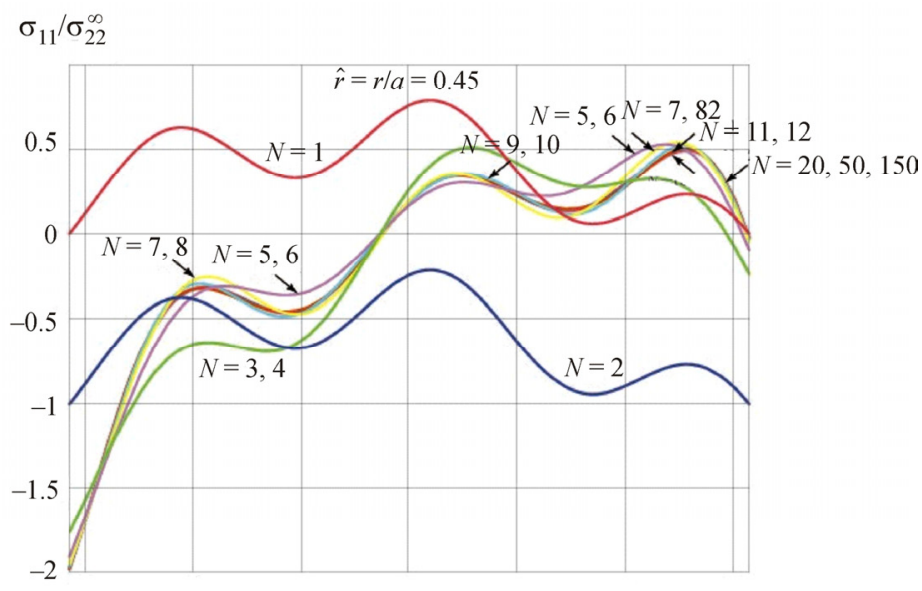

$b$

Fig. 29. The angular distribution of the stress tensor component $\sigma_{11}$ under mixed mode loading conditions $\left(\sigma_{11}^{\infty}, \sigma_{12}^{\infty}, \sigma_{22}^{\infty}\right)=(0,0.75,1) \sigma_{22}^{\infty}$ in different distances from the crack tip: $a$ ) for $\hat{r}=r / a=0.35 ; b)$ for $\hat{r}=r / a=0.45$

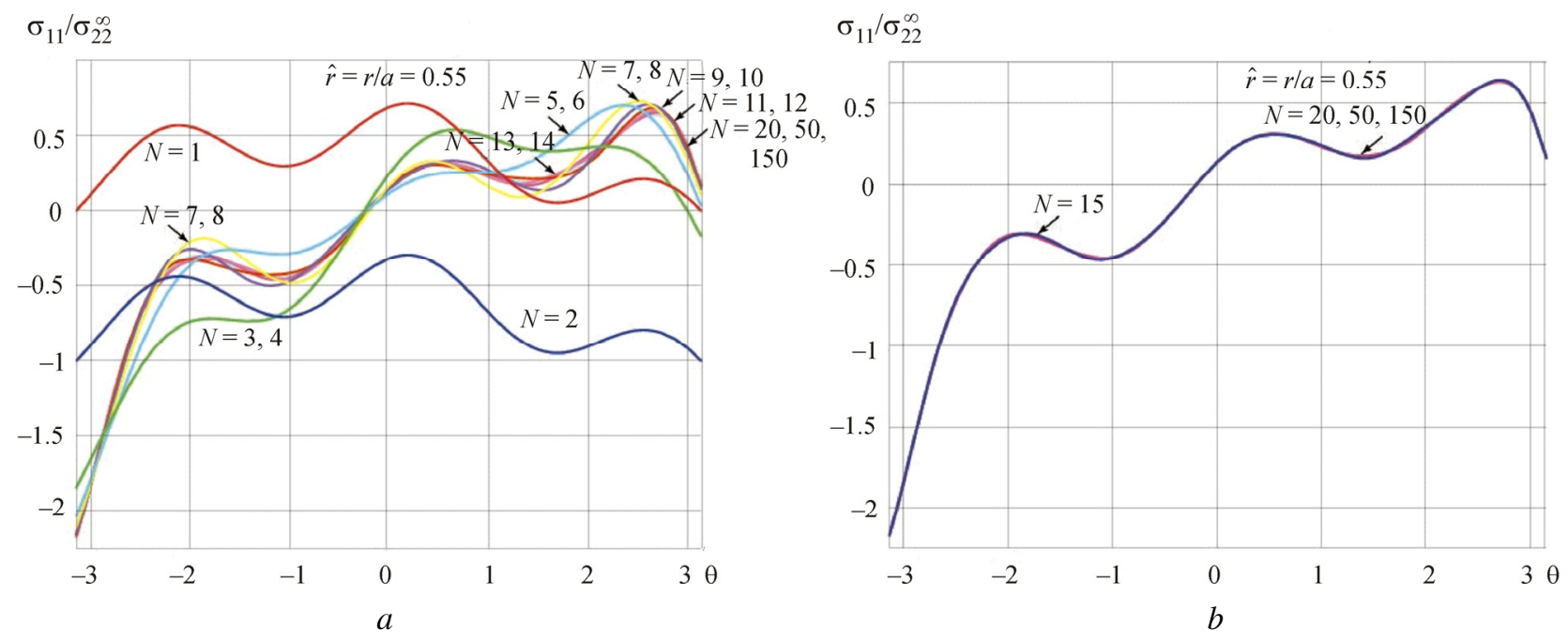

FIg. 30. The angular distributions of the stress tensor component $\sigma_{11}$ under mixed mode loading conditions $\left(\sigma_{11}^{\infty}, \sigma_{12}^{\infty}, \sigma_{22}^{\infty}\right)=(0,0.75,1) \sigma_{22}^{\infty}$, built with different numbers of held terms in asymptotic expansion 

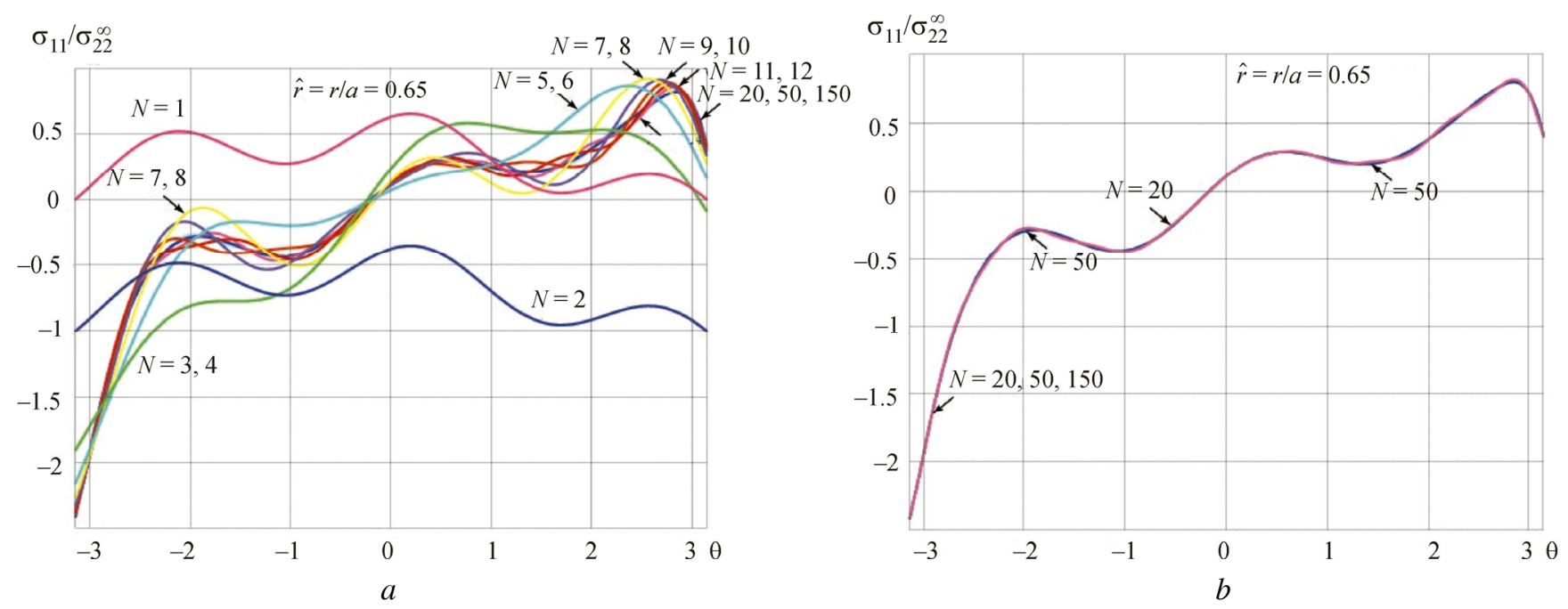

Fig. 31. The angular distributions of the stress tensor component $\sigma_{11}$ under mixed mode loading conditions $\left(\sigma_{11}^{\infty}, \sigma_{12}^{\infty}, \sigma_{22}^{\infty}\right)=(0,0.75,1) \sigma_{22}^{\infty}$, built with different numbers of held terms in asymptotic expansion

Fig. 27-31 demonstrate the angular distributions of the stress tensor components $\sigma_{22}$ and $\sigma_{11}$ under mixed mode loading conditions $\left(\sigma_{11}^{\infty}, \sigma_{12}^{\infty}, \sigma_{22}^{\infty}\right)=(0,0.75,1) \sigma_{22}^{\infty}$ in different distances from the crack tip. The figures clearly present that for more precise description of a stress field it is necessary to hold not only the T-stresses, but also the higher approximations in the complete asymptotic expansion in the vicinity of the crack tip.

\section{Conclusion and Results Discussion}

The work acquires analytic expressions for the amplitude coefficients of the complete stress field asymptotic expansion near the tips of two collinear cracks of finite length in the infinite plate medium in the conditions of 1) pure strain; 2) pure transverse shear; 3) mixed mode loading. It demonstrates that along with the main member of the asymptotic expansion and the T-stress, the higher approximations are major fracture mechanic parameters of the complete asymptotic expansion, the consideration of which is essential for precise evaluation of the stress fields near the fault tip, and, consequently, for more precise evaluation of the bearing capacity and the life duration of the specimen. The analytical expressions of the stress field asymptotic expansion coefficients for any previously given number of the held terms are only available for the plate medium with one crack of a finite length [17]; for this reason it is important to expand the class of the specimens providing analytical dependences of the amplitude coefficients on the applied load and the geometric parameters of specimens. The asymptotic solution found in the present paper for an infinite plate medium with two cracks of equal length may be used for: 1) checking the numerical processing of the experimental data obtained with polarization-optic methods of the deformed solid body mechanics for cracked specimens; 2) checking the numerical algorithms actively developed today for calculation of the multiparameter stress field amplitude coefficients $[6,9,37,43]$.

The project is developed with the financial support of the Russian Foundation for Basic Research (Grant No. 13-01-97009).

\section{References}

1. Wei R.P. Fracture Mechanics. Integration of Mechanics, Materials Science and Chemistry. Cambridge: Cambridge University Press, 2014. 232 p.

2. Voyiadjis G.Z. Handbook of damage mechanics: nano to macro scale for materials and structures. Berlin: Springer, 2015. $1577 \mathrm{p}$.

3 Gupta M., Alderliesten R.C., Benedictus R. A review of $\mathrm{T}$-stress and its effects in fracture. Engineering Fracture Mechanics, 2015, vol. 134, pp. 218-241.

4. Weibgraeber P., Becker W. Crack initiation at weak stress singularities - Finite Fracture Mechanics approach. Procedia Materials Science, 2014, no. 3, pp. 153-158.

5. Shlyannikov V.N., Zakharov A.P. Multiaxial crack growth under variable T-stress. Engineering Fracture Mechanics, 2014, vol. 123, pp. 86-99.

6. Berto F., Lazzarin P. Multiparametric full-field representations of the in-plane stress fields ahead of cracked components under mixed mode loading. International Journal of Fatigue, 2013, vol. 46, pp. 16-26.

7. Lu K., Meshii T. A systematic investigation of T-stresses for a variety of center-cracked tension specimen. Theoretical and Applied Fracture Mechanics, 2015, no. 2, pp. 74-81.

8. Akbardoost J., Rastin A. Comprehensive date for calculating the higher order terms of crack tip stress field in disk type specimens under mixed-mode loading. Theoretical and Applied Fracture Mechanics, 2015, vol. 76, pp. 75-90.

9. Akbardoost J., Ayatollahi M.R. Experimental analysis of mixed mode crack propagation in brittle rocks: The effect of nonsingular terms. Engineering Fracture Mechanics, 2014, vol. 129, pp. 77-89.

10. Berto F., Lazzarin P. On higher order terms in the crack tip stress field. International Journal of Fracture, 2010, vol. 161, pp. 221-226. 
11. Vesely V., Frantik P., Sobek J., Malikova L., Seitl S. Multi-parametric crack tip stress state description for evaluation of nonlinear zone width in silicate composite specimens in component splitting/ bending test geometry. Fatigue and Fracture of Engineering Materials and Structures, 2015, vol. 38, no. 2, pp. 200-214.

12. Vesely V., Sobek J., Sestakova L., Frantik P. Multiparametric crack tip stress state description for estimation of fracture process zone extent in silicate composite WST specimens. Frattura ed Intergrita Strutturale, 2013, vol. 25, pp. 69-78.

13. Malikova L., Vesely V. Williams expansion terms and their importance for accurate stress field description in specimens with a crack. Transaction of the VSB- Technical University of Ostrava, Mechanical Series, 2013, no. 2, vol. LIX, pp. 109-114.

14. Ayatollahi M.R., Nejati M. An over-deterministic method for calculation of coefficients of crack tip asymptotic field from finite element analysis. Fatigue and Fracture of Engineering Materials and Structures, 2010, vol. 34, pp. 159-176.

15. Sevecek O., Bermejo R., Profant T., Kotoul M. Influence of the T-stress on the crack bifurcation phenomenon in ceramic laminates. Procedia Materials Science, 2014, vol. 3, pp. 1062-1067.

16. Malikova L., Vesely V. Significance of higher-order terms of the Williams expansion for plastic zone extent estimation demonstrated on a mixed-mode geometry. Procedia Materials Science, 2014, vol. 3, pp. 1383-1388.

17. Hello G., Tahar M.B., Roelandt J.-M. Analytical determination of coefficients in crack-tip stress expansions for a finite crack in an infinite plane medium. International Journal of Solids and Structures, 2012, vol. 49, pp. 556-566.

18. Sestakova L. Using the multi-parameter fracture mechanics for more accurate description of stress and displacement crack tip fields. Key Engineering Materials, 2013, vol. 586, pp. 237-240.

19. Sestakova L. How to enhance efficiency and accuracy of the over-deterministic method used for determination of the coefficients of the higher-order terms in Williams expansion. Applied Mechanics and materials, 2013, vol. 245, pp. 120-125.

20. Stepanova L.V., Fedina M.Ye. Self-similar solution of a tensile crack problem in a coupled formulation. Journal of Applied Mathematics and Mechanics, 2008, vol. 72(3), pp. 360-368.

21. Stepanova L.V., Igonin S.A. Perturbation method for solving the nonlinear eigenvalue problem arising from fatigue crack growth problem in a damaged medium. Applied Mathematical Modelling, 2014, vol. 38 (14), pp. 3436-3455.

22. Stepanova L.V., Adylina E.M. Stress-strain state in the vicinity of a crack tip under mixed loading. Journal of Applied Mechanics and Technical Physics, 2014, vol. 55(5), pp. 885-895.

23. Beliakova T.A., Kulagin V.A. The eigenspectrum approach and T-stress at the mixed - mode crack tip for a stress state dependent material. Procedia Materials Science, 2014, vol. 3, pp. 147-152.

24. Stepanova L.V., Yakovleva E.M. Mixed-mode loading of the cracked plate under plane stress conditions. PNRPU Mechanics Bulletin, 2014, no. 3, pp. 129-162. DOI: 10.15593/perm.mech/2014.3.08

25. Stepanova L., Yakovleva E., Mironova E. Asymptotic self-similar solution of the creep crack problems in damaged materials under mixed mode loading. Applied Mechanics and Materials, 2015, vol. 784, pp. 145-152.
26. Stepanova L.V. Eigenspectra and orders of stress singularity at a mode I crack tip for a powerlaw medium. Comptes Rendus - Mecanique, 2008, vol. 336 (1-2), pp. 232-237.

27. Stepanova L.V. Eigenvalue analysis for a crack in a power-law material. Computational Mathematics and Mathematical Physics, 2009, vol. 49(8), pp. 1332-1347.

28. Shlyannikov V.N., Tumanov A.V., Zakharov A.P. The mixed mode crack growth rate in cruciform specimens subject to biaxial loading. Theoretical and Applied Fracture Mechanics, 2014, vol. 73, pp. 68-81.

29. Profant T., Klusak J., Sevecek O., Kotoul M., Hrstka M., Marcian P. An effect of the first nonsingular term of the Williams asymptotic expansion to the stability of the bi-material orthotropic notch. Key Engineering Materials, 2014, vol. 592-593, pp. $745-748$

30. Matvienko Yu.G. Nesinguliarnye T-napriazheniia $\mathrm{v}$ problemakh dvukhparametricheskoi mekhaniki razrusheniia [Nonsingular T-stress in problems of two-parameter fracture mechanics]. Zavodskaia laboratoriia. Diagnostika materialov, 2012, vol. 78, no. 2, pp. 51-58.

31. Matvienko Yu.G. Nesinguliarnye T-napriazheniia v kriteriiakh mekhaniki razrusheniia tel $\mathrm{s}$ treshchinami [The nonsingular T-stress in fracture mechanics criteria of solids with notches]. Vestnik Nizhegorodskogo universiteta, 2011, no. 4-5, pp. 2651-2652.

32. Litvinov I.A., Matvienko Yu.G., Razumovsky I.A. O tochnosti opredeleniia nesinguliarnykh komponent tenzora naprjazhenij $\mathrm{v}$ vershine treshhiny $\mathrm{s}$ primeneniem metoda jekstrapoljacii [On the accuracy of determination of nonsingular component in stress field at crack tip using extrapolation method]. Mashinostroenie $i$ inzhenernoe obrazovanie. 2014, no. 2 (39), pp. 47-52.

33. Matvienko Y.G., Pochinkov R.A. Effect of nonsingular T-stress components on the plasticdeformation zones near the tip of a mode I crack. Russian metallurgy (Metally), 2013, vol. 2013, no. 4, pp. 262-271.

34. Pisarev V.S., Matvienko Y.G., Odintsev I.N. Opredelenie parametrov mekhaniki razrusheniia pri malom prirashchenii dliny treshchiny [Determination of fracture Mechanics Parametera at a small increment of the crack length]. Zavodskaia laboratoriia. Diagnostika materialov, 2012, vol. 78, no. 4, pp. 45-51.

35. Matvienko Y.G. [Two approaches to accounting the nonsingular T-stress in fracture mechanics criteria for cracked bodies]. Problemy mashinostroeniia $i$ nadezhnosti mashin, 2011, no. 5, pp. 104-110.

36. Matvienko Y.G. Dvuhkparametricheskaia mekhanika razrusheniia $\mathrm{v}$ sovremennykh problemakh prochnosti [Two parametric fracture mechanics in actual reliability problems]. Problemy mashinostroeniia i nadezhnosti mashin, 2013, no. 5, pp. 37-46.

37. Matvienko Y.G., Chernyatin A.S., Razumovsky I.A. Chislennyi analiz nesinguliarnykh sostavliaiushchikh trekhmernogo polia napriazhenii $\mathrm{v}$ vershine treshchiny smeshannogo tipa [Numerical analysis of non-singular stress tensor components in the vicinity of the mixed-mode crack tip]. Problemy mashinostroeniia i nadezhnosti mashin, 2013, no. 4, pp. 40-48.

38. Matvienko Yu. G. The effect of the non-singular T-stress components on crack tip plastic flow zone under Mode I. Procedia Materials Science, 2014, vol. 3, pp. 141-146. 
39. Williams M.L. On the stress distribution at the base of a stationary crack. Journal of Applied Mechanics, 1957, vol. 24, pp. 109-114.

40. Kolosoff G. Primenenie kompleksnoi peremennoi k teorii uprugosti [Application of the complex variable to the elasticity theory]. Moscow, Leningrad: 1935, 224 p.

41. Muskhelishvili N.I Nekotorye osnovnye zadachi matematicheskoi teorii uprugosti [Some basic problems of mathematical problems of elasticity theory]. Moscow: Nauka, 1966, 708 p.
42. Mirsayar M.M., Aliha M.R.M., Samaei A.T. On fracture initiation angle near bi-material notches - Effects of first nonsingular terms. Engineering fracture mechanics, 2014, vol. 119, pp. 124-131.

43. Kotousov A., Lazzarin P., Berto F., Pook L.P. Threedimensional stress state at crack tip induced by shear and antiplane loading. Engineering Fracture Mechanics, 2013, vol. 108, pp. 65-74.

Original Russian Text:

Stepanova L.V., Roslyakov P.S. Complete asymptotic expansion M. Williams near the crack tips of collinear cracks of equal lengths in an infinite plane medium. PNRPU Mechanics Bulletin. 2015, no. 4, pp. 188-225. DOI: 10.15593/perm.mech/2015.4.12 